\title{
Thermodynamic equilibrium of massless fermions with vorticity, chirality and electromagnetic field
}

\author{
Matteo Buzzegoli
}

\begin{abstract}
We present a study of the thermodynamics of the massless free Dirac field at equilibrium with axial charge, angular momentum and external electromagnetic field to assess the interplay between chirality, vorticity and electromagnetic field in relativistic fluids. After discussing the general features of global thermodynamic equilibrium in quantum relativistic statistical mechanics, we calculate the thermal expectation values. Axial imbalance and electromagnetic field are included nonperturbatively by using the exact solutions of the Dirac equation, while a perturbative expansion is carried out in thermal vorticity. It is shown that the chiral vortical effect and the axial vortical effect are not affected by a constant homogeneous electromagnetic field.
\end{abstract}

\section{Introduction}

The collective macroscopic behavior of matter in the presence of quantum anomalies and external fields is an increasingly important subject in several fields of physics. Specifically, the experiments of relativistic heavy-ion collisions at RHIC and LHC have posed new and interesting questions about the theoretical foundations of relativistic collective phenomena. The experimental data of heavy ion collisions indicates the creation of a deconfined quark gluon plasma in a strongly coupled regime at extreme conditions of temperature, density, thermal vorticity [1] and magnetic fields [2]. Moreover, it was argued [3, 4] that the fluctuations of topological configurations of the QCD vacuum in the early stages of a heavy ion collision generate a chiral imbalance, which is an imbalance between the number of right- and left- handed quarks. Despite the usual relativistic hydrodynamic has been very effective [5] in reproducing the experimental data for collective flow phenomena, it is

Matteo Buzzegoli

Universitá di Firenze and INFN Sezione di Firenze, Via G. Sansone 1, I-50019 Sesto Fiorentino

(Firenze), Italy, e-mail: buzzegoli@fi .infn.it 
now essential for the interpretation of heavy ion collisions to address hydrodynamics in the contemporaneous presence of chiral imbalance, thermal vorticity, and external electromagnetic fields.

The first crucial step towards understanding the hydrodynamics of matter subject to external fields is to study its thermodynamic properties. It is the main purpose of this contribution to investigate the effects of an external electromagnetic field on the thermodynamics of a chiral vorticous fluid. The effects of electromagnetic fields on (non-chiral non-vorticous) relativistic quantum fluids were already studied in the past, see for instance [6,7] and reference therein and [8] for the special case of a constant magnetic field. More recently this topic has been addressed in [9] using the Zubarev's non-equilibrium statistical operator, in [10] using the generating functional method and in $[11,12,13]$ with Wigner function derived from kinetic theory.

This contribution aims to highlight the modifications caused by chiral imbalance and by thermal vorticity. The paper is organized in the following way. In Sec. 2, we introduce the global thermal equilibrium of a chiral system with the contemporaneous presence of an external electromagnetic field and a thermal vorticity within Zubarev's non-equilibrium statistical operator formalism. In Sec. 2.1 we give a brief overview of the main results for the case of a chiral Dirac field in the absence of the electromagnetic field. In Sec. 3 we review the relativistic quantum theory of fermions under the effect of an external magnetic field. Then, we obtain the exact form of the chiral fermionic propagator with external constant magnetic field and we obtain the exact thermal averages of the axial and electric currents. In Sec. 5 we examine the properties of a system at thermal equilibrium with constant vorticity and electromagnetic field. The last part of the paper is concerned with the consequences of an electromagnetic field on the chiral vortical effect and the axial vortical effect.

\section{Notation}

In this work we use the natural unit system in which $\hbar=c=G=k_{B}=1$. The Minkowski metric is defined by the tensor $\eta_{\mu \nu}=\operatorname{diag}(1,-1,-1,-1)$; for the Levi-Civita symbol we use the convention $\epsilon^{0123}=+1$.

Operators in Hilbert space will be denoted by a large upper hat, e.g. $\widehat{T}$ (with the exception of Dirac field operator that is denoted by $\Psi$ ). The stress-energy tensor used to define Poincaré generators is always assumed to be symmetric with an associated vanishing spin tensor. 


\section{General global equilibrium with electromagnetic field}

In this section we introduce the methods to study the thermodynamic equilibrium of a quantum relativistic system in the presence of a chiral imbalance and of an external electromagnetic field. For that purpose we review the Zubarev method of stationary non-equilibrium density operator $[14,15]$ (see also $[16,17,18,19]$ for recent developments) and we discuss the inclusion of a conserved axial current and of an external electromagnetic field.

When we are dealing with a relativistic system we must consider local quantities in order to address the appropriate covariant properties. To identify those quantities we use a Arnowitt-Deser-Misner (ADM) decomposition of space-time [14, 15, 16, 17]. Choose then a foliation of space-time and suppose that the system in consideration thermalize faster than the evolution of "time" $\tau$ in which we are interested. At each step of evolution $\mathrm{d} \tau$, the system is at local thermal equilibrium and the macroscopic behavior of the system is described by a stress-energy density $T_{\mu \nu}(x)$, an (electric) current density $j_{\mu}(x)$ and an axial current $j_{\mathrm{A} \mu}(x)$, all lying on a space-like hypersurface $\Sigma(\tau)$. We can then describe the thermal properties of the system with a density operator which lives on $\Sigma(\tau)$. As in the non-relativistic case, the density operator at local equilibrium $\widehat{\rho}_{\text {LE }}$ is obtained as the operator which maximizes the entropy $S=-\operatorname{tr}(\widehat{\rho} \log \widehat{\rho})$. To reproduce the actual thermodynamics on the hyper-surface we maximize the entropy with the constraints that the mean values of the stress-energy tensor and of the currents on $\Sigma(\tau)$ corresponds to the values of the densities $T_{\mu \nu}(x)$, $j_{\mu}(x)$ and $j_{\mathrm{A} \mu}(x)$ [15]. To obtain these densities we project the stress-energy tensor and the current mean values onto $n$, i.e. the normalized four-vector perpendicular to $\Sigma$ :

$$
\begin{gathered}
n_{\mu}(x) \operatorname{tr}\left[\widehat{\rho} \widehat{T}^{\mu v}(x)\right]=n_{\mu}(x)\left\langle\widehat{T}^{\mu v}(x)\right\rangle \equiv n_{\mu}(x) T^{\mu v}(x), \\
n_{\mu}(x) \operatorname{tr}\left[\widehat{\rho} \widehat{j}^{\mu}(x)\right]=n_{\mu}(x)\left\langle\widehat{j}^{\mu}(x)\right\rangle \equiv n_{\mu}(x) j^{\mu}(x),
\end{gathered}
$$

and similarly for the axial current. We could also impose a constraint on the angular momentum density, but since we are choosing the Belinfante operator as the stress-energy tensor, it turns out that this additional requirement is automatically taken into account [20].

The maximum solution $\widehat{\rho}_{\text {LE }}$ gives the Local Equilibrium Density Operator (LEDO) [16, 17]:

$$
\widehat{\rho}_{\mathrm{LTE}}=\frac{1}{Z} \exp \left[-\int_{\Sigma} \mathrm{d} \Sigma_{\mu}\left(\widehat{T}^{\mu v}(x) \beta_{v}(x)-\zeta(x) \widehat{j}^{\mu}(x)-\zeta_{\mathrm{A}}(x) \widehat{j}_{\mathrm{A}}^{\mu}(x)\right)\right],
$$

where $\beta^{\mu}$ is the four-temperature vector such that $T=1 / \sqrt{\beta^{2}}$ is the proper comoving temperature, $\zeta$ and $\zeta_{\mathrm{A}}$ are the ratio of comoving chemical potentials and the temperature (e.g. $\zeta=\mu / T$ ) and $Z$ is the partition function. In the presence of an external electromagnetic field, we indicate with $A^{\mu}(x)$ the non-dynamical gauge field and with $F^{\mu \nu}=\partial^{\mu} A^{v}-\partial^{v} A^{\mu}$ the electromagnetic strength tensor. Therefore, 
the operator relations stemming for conservation equations are

$$
\partial_{\mu} \widehat{j}^{\mu}=0, \quad \partial_{\mu} \widehat{T}^{\mu \nu}=\widehat{j}_{\lambda} F^{\nu \lambda}, \quad \partial_{\mu} \widehat{j}_{\mathrm{A}}^{\mu}=0 .
$$

Furthermore, the four-momentum operator $\widehat{P}$ and the conserved charges $\widehat{Q}_{i}$ are obtained by

$$
\widehat{P}^{\mu}=\int_{\Sigma} \mathrm{d} \Sigma_{\lambda} \widehat{T}^{\lambda \mu}, \quad \widehat{Q}_{i}=\int_{\Sigma} \mathrm{d} \Sigma_{\lambda}{\widehat{j_{i}}}^{\lambda},
$$

while the angular momentum is

$$
\widehat{J}^{\mu \nu}=\int_{\Sigma} \mathrm{d} \Sigma_{\lambda}\left(x^{\mu} \widehat{T}^{\lambda v}-x^{\nu} \widehat{T}^{\lambda \mu}\right) .
$$

Notice that, as we will discuss in details in Sec. 3, the four-momentum $\widehat{P}$ and the angular momentum $\widehat{J}$ in the presence of external electromagnetic field are neither conserved nor the generators of translations and Lorentz transformations.

In the case of Dirac fermions interacting with an external gauge field, the explicit form of the operators above is

$$
\begin{aligned}
\widehat{j}^{\mu} & =q \bar{\Psi} \gamma^{\mu} \Psi, \quad \widehat{j}_{\mathrm{A}}^{\mu}=\bar{\Psi} \gamma^{\mu} \gamma^{5} \Psi \\
\widehat{T}^{\mu \nu} & =\frac{\mathrm{i}}{4}\left[\bar{\Psi} \gamma^{\mu} \vec{\partial}^{v} \Psi-\bar{\Psi} \gamma^{\mu} \overleftarrow{\partial}^{v} \Psi+\bar{\Psi} \gamma^{\nu} \vec{\partial}^{\mu} \Psi-\bar{\Psi} \gamma^{\nu} \overleftarrow{\partial}^{\mu} \Psi\right]-\frac{1}{2}\left(\widehat{j}^{\mu} A^{v}+\widehat{j}^{\nu} A^{\mu}\right)
\end{aligned}
$$

and the stress-energy tensor and the electric current indeed satisfy the relations (2). Regarding the axial current $\widehat{j_{\mathrm{A}}}$, we also have to take into account the chiral anomaly. The chiral anomaly affects the axial current divergence as follows

$$
\partial_{\mu} \widehat{j}_{\mathrm{A}}^{\mu}=-\frac{1}{8} \epsilon^{\mu \nu \rho \lambda} \frac{q^{2}}{2 \pi^{2}} F_{\mu \nu} F_{\rho \lambda}=-\frac{q^{2}}{2 \pi^{2}}(E \cdot B),
$$

where $q$ is the electric charge of the fermion, and $E$ and $B$ are comoving electric and magnetic field, defined by

$$
F^{\mu \nu}=E^{\mu} u^{v}-E^{v} u^{\mu}-\epsilon^{\mu \nu \rho \sigma} B_{\rho} u_{\sigma},
$$

with $u$ the fluid velocity. Even when the product $E \cdot B$ is non-vanishing and consequently the axial current is not conserved, we can still define a new conserved "axial" current by means of the Chern-Simons current $K$, whose divergence gives the chiral anomaly:

$$
K^{\mu}=\epsilon^{\mu \nu \rho \sigma} A_{\nu} F_{\rho \sigma}, \quad \frac{q^{2}}{8 \pi^{2}} \partial_{\mu} K^{\mu}=\frac{q^{2}}{2 \pi^{2}}(E \cdot B) .
$$

The new conserved axial current $\widehat{j}_{C S}$ is then defined as 


$$
\widehat{j}_{\mathrm{CS}}^{\mu}=\widehat{j}_{\mathrm{A}}^{\mu}+\frac{q^{2}}{8 \pi^{2}} K^{\mu}, \quad \partial_{\mu} \widehat{j}_{\mathrm{CS}}^{\mu}=0,
$$

and the axial chemical potential $\mu_{\mathrm{A}}$ is to be associated to this current. Since the additional current $K$ depends only on external fields, it is not a quantum operator and it does not contribute to thermal averages. Therefore, all the results discussed in the absence of chiral anomaly will also be valid for the case of equilibrium with conserved Chern-Simons current. Because there is no difference in the results, we will continue to denote the current associated to $\mu_{\mathrm{A}}$ inside the statistical operator with $\widehat{j_{\mathrm{A}}}$ even when the chiral anomaly is non-vanishing.

Let us now move to describe the system at global thermal equilibrium. The global equilibrium is reached when the statistical operator (1) is time independent. This occurs when the integrand inside Eq. (1) is divergenceless [21]. Then, it is easily proven using relations (2) that global thermal equilibrium is realized when the following relations are satisfied:

$$
\partial_{\mu} \beta_{\nu}(x)+\partial_{\nu} \beta_{\mu}(x)=0, \quad \partial^{\mu} \zeta(x)=F^{\nu \mu} \beta_{\nu}(x), \quad \partial^{\mu} \zeta_{\mathrm{A}}(x)=0 .
$$

The inverse four-temperature and the axial chemical potential solves the previous conditions if they are given by [16]:

$$
\beta_{\mu}(x)=b_{\mu}+\varpi_{\mu \rho} x^{\rho}, \quad \zeta_{\mathrm{A}}=\text { constant }
$$

where $b$ is a constant time-like four-vector and $\varpi$ is a constant anti-symmetric tensor. We refer to $\varpi$ as the thermal vorticity because it is the anti-symmetric derivative of inverse four-temperature:

$$
\varpi_{\mu \nu}=-\frac{1}{2}\left(\partial_{\mu} \beta_{\nu}-\partial_{\nu} \beta_{\mu}\right)
$$

and because it contains information about the fluid's acceleration and rotation. Indeed, if the $\beta$ four-vector is a time-like vector, then we can choose the $\beta$-frame as hydrodynamic frame $[21,10]$. The unitary four-vector fluid velocity $u$ is therefore identified with the direction of $\beta$ :

$$
u^{\mu}(x)=\frac{\beta^{\mu}(x)}{\sqrt{\beta_{\rho}(x) \beta^{\rho}(x)}} .
$$

As long as we are considering physical observables in a region where the coordinate $x$ are such that $\beta(x)$ is a time-like vector, this definition provides a proper choice for the fluid velocity. We can decompose the thermal vorticity into two space-like vector fields, each having three independent components, by projecting along the time-like fluid velocity $u$ :

$$
\varpi^{\mu \nu}=\epsilon^{\mu \nu \rho \sigma} w_{\rho} u_{\sigma}+\alpha^{\mu} u^{\nu}-\alpha^{\nu} u^{\mu} .
$$

The four-vectors $\alpha$ and $w$ are explicitly written inverting the previous relation: 


$$
\alpha^{\mu}(x) \equiv \varpi^{\mu v} u_{v}, \quad w^{\mu}(x) \equiv-\frac{1}{2} \epsilon^{\mu v \rho \sigma} \varpi_{v \rho} u_{\sigma} .
$$

The vectors $\alpha$ and $w$ depend on the coordinates, are space-like, and are orthogonal to $u$. All the quantity $u, \varpi, \alpha, w$ are dimensionless. From their definitions, we can easily show that $\alpha$ and $w$ are given by

$$
\alpha^{\mu}=\sqrt{\beta^{2}} a^{\mu}, \quad w^{\mu}=\sqrt{\beta^{2}} \omega^{\mu},
$$

where $a$ and $\omega$ are the local acceleration and rotation of the fluid, which are given by

$$
a^{\mu}=u_{v} \partial^{v} u^{\mu}, \quad \omega^{\mu} \equiv \frac{1}{2} \epsilon^{\mu v \rho \sigma} u_{\sigma} \partial_{v} u_{\rho}
$$

Furthermore, it will proves useful to define the projector into the orthogonal space of fluid velocity:

$$
\Delta_{\mu \nu} \equiv g_{\mu \nu}-u_{\mu} u_{v}
$$

and the four vector $\gamma$ orthogonal to the other ones: $u, \alpha, w$

$$
\gamma^{\mu}=\epsilon^{\mu \nu \rho \sigma} w_{\nu} \alpha_{\rho} u_{\sigma}=(\alpha \cdot \varpi)_{\lambda} \Delta^{\lambda \mu} .
$$

The $\varpi$ decomposition above defines a tetrad $\{u, \alpha, w, \gamma\}$ which can be used as a basis for four-vectors. It must be noticed, however, that the tetrad is neither unitary nor orthonormal, indeed in general we have $\alpha \cdot w \neq 0$.

Returning to the global equilibrium conditions (5), notice that in the absence of electromagnetic field also $\zeta$ must be a constant. In that case the global equilibrium statistical operator takes the following form $[22,23]$ :

$$
\widehat{\rho}=\frac{1}{Z} \exp \left\{-b \cdot \widehat{P}+\frac{1}{2} \varpi: \widehat{J}+\zeta \widehat{Q}+\zeta_{\mathrm{A}} \widehat{Q}_{\mathrm{A}}\right\} .
$$

The thermodynamics of Dirac fermions which follows from this operator is quickly reviewed in Section 2.1

For the case of a non-vanishing electromagnetic field instead, we need to solve the equation:

$$
\partial^{\mu} \zeta(x)=F^{\sigma \mu} \beta_{\sigma} .
$$

To find the solution, we first derive it respect to $\partial^{v}$ :

$$
\partial^{v} \partial^{\mu} \zeta=\partial^{v}\left(F^{\sigma \mu} \beta_{\sigma}\right)
$$

Since we can exchange the order of the derivatives $\partial^{\nu} \partial^{\mu}$ on the 1.h.s. of (8), it follows that the anti-symmetrization respect to indices $\mu$ and $v$ of (8) must be vanishing:

$$
\begin{aligned}
\partial^{v} \partial^{\mu} \zeta-\partial^{\mu} \partial^{v} \zeta & =0=\left[\partial^{v}\left(F^{\sigma \mu} \beta_{\sigma}\right)-\partial^{\mu}\left(F^{\sigma v} \beta_{\sigma}\right)\right] \\
& =\left[\beta_{\sigma}\left(\partial^{v} F^{\sigma \mu}-\partial^{\mu} F^{\sigma v}\right)+\left(\partial^{v} \beta_{\sigma}\right) F^{\sigma \mu}+\left(\partial^{\mu} \beta_{\sigma}\right) F^{v \sigma}\right] .
\end{aligned}
$$

Using the first Bianchi identity $\partial^{v} F^{\sigma \mu}+\partial^{\mu} F^{v \sigma}+\partial^{\sigma} F^{\mu v}=0$, we obtain 


$$
\beta_{\sigma} \partial^{\sigma} F^{\mu v}+\left(\partial^{v} \beta_{\sigma}\right) F^{\mu \sigma}+\left(\partial^{\mu} \beta_{\sigma}\right) F^{\sigma v}=0 .
$$

We may recognize the Lie derivative of $F$ along $\beta$ in the previous equation. This constitutes a first condition for global equilibrium, the system can reach global equilibrium only when

$$
\mathcal{L}_{\beta}(F)=0, \quad \leftrightarrow \quad \beta_{\sigma}(x) \partial^{\sigma} F^{\mu \nu}(x)=\varpi^{\mu}{ }_{\sigma} F^{\sigma v}(x)-\varpi^{\nu}{ }_{\sigma} F^{\sigma \mu}(x),
$$

that is to say when the electromagnetic field follows the field lines of inverse-four temperature.

To actually solve Eq. (7) we translate the global equilibrium condition of the strength tensor (9) to the four-vector potential $A^{\mu}$. We see that the constraint (9) is satisfied if $A$ solves

$$
\beta_{\sigma}(x) \partial^{\sigma} A^{\mu}(x)=\varpi_{\sigma}^{\mu} A^{\sigma}(x)+\partial^{\mu} \Phi(x),
$$

where $\Phi$ is a smooth function of $x$. In ref. [18] was also stated that a gauge potential with vanishing Lie derivative along $\beta$ gives a stationary statistical operator, which is condition (10). It is important to stress that after a gauge transformation the condition (10) still holds true for the new gauge potential because the function $\Phi$ is also affected by the gauge transformation. Indeed, let $A^{\mu}$ satisfies Eq. (10); after the gauge transformation $A^{\prime \mu}=A^{\mu}+\partial^{\mu} \Lambda$ we find:

$$
\begin{aligned}
\beta_{\sigma} \partial^{\sigma} A^{\prime \mu} & =\beta_{\sigma} \partial^{\sigma} A^{\mu}+\beta_{\sigma} \partial^{\sigma} \partial^{\mu} \Lambda=\omega_{\sigma}^{\mu} A^{\sigma}+\partial^{\mu} \Phi+\partial^{\mu}\left(\beta_{\sigma} \partial^{\sigma} \Lambda\right)-\left(\partial^{\mu} \beta_{\sigma}\right) \partial^{\sigma} \Lambda \\
& =\varpi^{\mu}{ }_{\sigma}\left(A^{\sigma}+\partial^{\sigma} \Lambda\right)+\partial^{\mu}\left(\Phi+\beta_{\sigma} \partial^{\sigma} \Lambda\right)=\varpi^{\mu}{ }_{\sigma} A^{\prime \sigma}+\partial^{\mu} \Phi^{\prime}
\end{aligned}
$$

which is exactly condition (10) for $A^{\prime \mu}$ and for $\Phi^{\prime}$, that is $\Phi$ shifted by the transport of $\Lambda$ along $\beta$.

We can now write Eq. (7) by taking advantage of Eq. (10):

$$
\begin{aligned}
\partial^{\mu} \zeta & =F^{\sigma \mu} \beta_{\sigma}=\beta_{\sigma}\left(\partial^{\sigma} A^{\mu}-\partial^{\mu} A^{\sigma}\right)=\beta_{\sigma} \partial^{\sigma} A^{\mu}-\partial^{\mu}\left(\beta_{\sigma} A^{\sigma}\right)+\left(\partial^{\mu} \beta_{\sigma}\right) A^{\sigma} \\
& =\varpi_{\sigma}^{\mu} A^{\sigma}+\partial^{\mu} \Phi-\varpi_{\sigma}^{\mu} A^{\sigma}-\partial^{\mu}\left(\beta_{\sigma} A^{\sigma}\right) .
\end{aligned}
$$

We can then collect all the derivatives together into the equation

$$
\partial^{\mu}\left(\zeta-\Phi+\beta_{\sigma} A^{\sigma}\right)=0,
$$

from which we immediately get the solution:

$$
\zeta(x)=\zeta_{0}-\beta_{\sigma}(x) A^{\sigma}(x)+\Phi(x),
$$

where $\zeta_{0}$ is a constant. The parameter $\Phi$ is analogous to the parameter which grants gauge invariance to chemical potential in [24]. Even though Eq. (11) is given in terms of the gauge potential it is still gauge invariant. Indeed, we have shown that with a gauge transformation, $A^{\mu}$ and $\Phi$ transform as

$$
A^{\prime \mu}=A^{\mu}+\partial^{\mu} \Lambda, \quad \Phi^{\prime}=\Phi+\beta_{\sigma} \partial^{\sigma} \Lambda,
$$


therefore, the chemical potential $\zeta$ is overall unaffected by gauge transformations:

$$
\zeta(x)^{\prime}=\zeta_{0}-\beta_{\sigma}(x) A^{\prime \sigma}+\Phi^{\prime}=\zeta_{0}-\beta_{\sigma}(x) A^{\sigma}+\Phi-\beta_{\sigma} \partial^{\sigma} \Lambda+\beta_{\sigma} \partial^{\sigma} \Lambda=\zeta(x) .
$$

The global equilibrium statistical operator is then obtained from the local one in Eq. (1) by replacing the global equilibrium form of the thermodynamic fields $\beta, \zeta, \zeta_{\mathrm{A}}$ :

$$
\begin{aligned}
\widehat{\rho}= & \frac{1}{Z} \exp \left\{-\int_{\Sigma} \mathrm{d} \Sigma_{\mu}\left[\left(\widehat{T}^{\mu \nu}(x)+\widehat{j}^{\mu}(x) A^{\nu}(x)\right) \beta_{\nu}(x)\right.\right. \\
& \left.\left.-\left(\zeta_{0}+\Phi(x)\right) \widehat{j}^{\mu}(x)-\zeta_{\mathrm{A}} \widehat{j}_{\mathrm{A}}^{\mu}(x)\right]\right\} .
\end{aligned}
$$

This operator, on par with (6), is given in terms of global conserved quantities. The difference is that for the general form of the external magnetic field satisfying the constraint (9), the integration over the hyper-surface $\Sigma$ does not give easily recognizable quantities like the four-momentum and the angular momenta in Eq. (6). However, the identification of global conserved operators can be carried out in the special case of constant homogeneous electromagnetic field and it is discussed in the following sections.

\subsection{Vanishing electromagnetic field}

Before proceeding with the effects of electromagnetic fields, we briefly review the thermodynamics properties of a relativistic system in the presence of thermal vorticity but without an external electromagnetic field. Regarding thermal equilibrium in the presence of rotation, exact solutions for the free scalar and Dirac fields are discussed in $[25,26,27]$. Instead the effects of acceleration has been recently investigated using the Zubarev method in Ref. [27, 28, 29, 30, 31, 32]. Here we want to report the constitutive equations at second order on thermal vorticity discussed in [22,33] and in [23] including an axial current (see also [34] for first order in thermal vorticity and magnetic field). Using linear response theory on thermal vorticity, the thermal expectation value of a local operator $\widehat{O}(x)$ evaluated with statistical operator (9) can be written as [22, 23]:

$$
\begin{aligned}
\langle\widehat{O}(x)\rangle= & \langle\widehat{O}(0)\rangle_{\beta(x)}-\alpha_{\rho}\left\langle\left\langle\widehat{K}^{\rho} \widehat{O}\right\rangle\right\rangle-w_{\rho}\left\langle\left\langle\widehat{J}^{\rho} \widehat{O}\right\rangle\right\rangle+\frac{\alpha_{\rho} \alpha_{\sigma}}{2}\left\langle\left\langle\widehat{K}^{\rho} \widehat{K}^{\sigma} \widehat{O}\right\rangle\right\rangle \\
& +\frac{w_{\rho} w_{\sigma}}{2}\left\langle\left\langle\widehat{J}^{\rho} \widehat{J}^{\sigma} \widehat{O}\right\rangle\right\rangle+\frac{\alpha_{\rho} w_{\sigma}}{2}\left\langle\left\langle\left\{\widehat{K}^{\rho}, \widehat{J}^{\sigma}\right\} \widehat{O}\right\rangle\right\rangle+O\left(\varpi^{3}\right) .
\end{aligned}
$$

In the previous expression we indicated with double angular bracket the correlator

$$
\begin{aligned}
\left\langle\left\langle\widehat{K}^{\rho_{1}} \cdots \widehat{K}^{\rho_{n}} \widehat{J}^{\sigma_{1}} \ldots \widehat{J}^{\sigma_{m}}\right.\right. & \widehat{O}\rangle\rangle \equiv \int_{0}^{|\beta|} \frac{\mathrm{d} \tau_{1} \cdots \mathrm{d} \tau_{n+m}}{|\beta|^{n+m}} \times \\
& \times\left\langle\mathrm{T}_{\tau}\left(\widehat{K}_{-\mathrm{i} \tau_{1} u}^{\rho_{1}} \cdots \widehat{K}_{-\mathrm{i} \tau_{n} u}^{\rho_{n}} \widehat{J}_{-\mathrm{i} \tau_{n+1} u}^{\sigma_{1}} \cdots \widehat{J}_{-\mathrm{i} \tau_{n+m} u}^{\sigma_{m}} \widehat{O}(0)\right)\right\rangle_{\beta(x), c}
\end{aligned}
$$


where $\widehat{J}$ and $\widehat{K}$ are the comoving rotation and boost generators, identified by

$$
\widehat{K}^{\mu}=u_{\lambda} \widehat{J}^{\lambda \mu}, \quad \widehat{J}^{\mu}=\frac{1}{2} \epsilon^{\alpha \beta \gamma \mu} u_{\alpha} \widehat{J}_{\beta \gamma},
$$

and the averages $\langle\cdots\rangle_{\beta(x)}$ are evaluated at a fixed point $x$ with the homogeneous statistical operator

$$
\widehat{\rho}_{0}=\frac{1}{Z_{0}} \exp \left\{-\beta(x) \cdot \widehat{P}+\zeta(x) \widehat{Q}+\zeta_{\mathrm{A}}(x) \widehat{Q}_{\mathrm{A}}\right\} .
$$

The subscript $-\mathrm{i} \tau u$ indicates an imaginary translation along $u$ as follows

$$
J_{-\mathrm{i} \tau u}^{\mu} \equiv \mathrm{e}^{-\mathrm{i} \tau u \cdot \widehat{P}} \widehat{J}^{\mu} \mathrm{e}^{\mathrm{i} \tau u \cdot \widehat{P}} .
$$

Constitutive equations at second order on thermal vorticity of the stress-energy tensor, the electric current and the axial current can be obtained using the expansion in Eq. (13). We obtain [22, 33, 23]

$$
\begin{aligned}
\left\langle\widehat{T}^{\mu \nu}\right\rangle= & \mathbb{A} \epsilon^{\mu \nu \kappa \lambda} \alpha_{\kappa} u_{\lambda}+\mathbb{W}_{1} w^{\mu} u^{\nu}+\mathbb{W}_{2} w^{\nu} u^{\mu} \\
& +\left(\rho-\alpha^{2} U_{\alpha}-w^{2} U_{w}\right) u^{\mu} u^{v}-\left(p-\alpha^{2} D_{\alpha}-w^{2} D_{w}\right) \Delta^{\mu \nu} \\
& +A \alpha^{\mu} \alpha^{\nu}+W w^{\mu} w^{\nu}+G_{1} u^{\mu} \gamma^{\nu}+G_{2} u^{\nu} \gamma^{\mu}+O\left(\varpi^{3}\right), \\
\left\langle\widehat{j}_{\mathrm{V}}^{\mu}\right\rangle= & n_{\mathrm{V}} u^{\mu}+\left(\alpha^{2} N_{\alpha}^{\mathrm{V}}+w^{2} N_{\omega}^{\mathrm{V}}\right) u^{\mu}+W^{\mathrm{V}} w^{\mu}+G^{\mathrm{V}} \gamma^{\mu}+O\left(\varpi^{3}\right), \\
\left\langle\widehat{j}_{\mathrm{A}}^{\mu}\right\rangle= & n_{\mathrm{A}} u^{\mu}+\left(\alpha^{2} N_{\alpha}^{\mathrm{A}}+w^{2} N_{\omega}^{\mathrm{A}}\right) u^{\mu}+W^{\mathrm{A}} w^{\mu}+G^{\mathrm{A}} \gamma^{\mu}+O\left(\varpi^{3}\right) .
\end{aligned}
$$

Not all of these coefficients are independent, indeed conservation equations (2) impose the following relations [33] (this is explained in details in Sec. 5.2):

$$
\begin{aligned}
U_{\alpha} & =-|\beta| \frac{\partial}{\partial|\beta|}\left(D_{\alpha}+A\right)-\left(D_{\alpha}+A\right), \\
U_{w} & =-|\beta| \frac{\partial}{\partial|\beta|}\left(D_{w}+W\right)-D_{w}+2 A-3 W, \\
G_{1}+G_{2} & =2\left(D_{\alpha}+D_{w}\right)+A+|\beta| \frac{\partial}{\partial|\beta|} W+3 W,
\end{aligned}
$$

instead, for the first-order coefficients, conservation equations require that

$$
-2 \mathbb{A}=|\beta| \frac{\partial \mathbb{W}_{1}}{\partial|\beta|}+3 \mathbb{W}_{1}+\mathbb{W}_{2}
$$

For electric and axial current, we find that only the following equations must be fulfilled:

$$
|\beta| \frac{\partial W^{\mathrm{V}}}{\partial|\beta|}+3 W^{\mathrm{V}}=0, \quad|\beta| \frac{\partial W^{\mathrm{A}}}{\partial|\beta|}+3 W^{\mathrm{A}}=0
$$


We can also take advantage of the Lorentz symmetry to show that the thermal coefficients $\mathbb{S}$ and $\Gamma_{w}$ of the canonical spin tenor constitutive equation:

$\left\langle\frac{\mathrm{i}}{8} \bar{\Psi}\left\{\gamma^{\lambda},\left[\gamma^{\mu}, \gamma^{\nu}\right]\right\} \Psi\right\rangle=\mathbb{S} \epsilon^{\lambda \mu \nu \rho} u_{\rho}+\Gamma_{w}\left(u^{\lambda} \varpi^{\mu \nu}+u^{\nu} \varpi^{\lambda \mu}+u^{\mu} \varpi^{\nu \lambda}\right)+O\left(\varpi^{2}\right)$,

satisfy the following relations

$$
\begin{aligned}
-\left(\frac{\mathbb{S}}{|\beta|}+\frac{\partial \mathbb{S}}{\partial|\beta|}\right) & =2 \mathbb{A}^{\mathrm{Can}}, \\
2 \frac{\mathbb{S}}{|\beta|} & =\mathbb{W}_{1}^{\mathrm{Can}}-\mathbb{W}_{2}^{\mathrm{Can}}, \\
\frac{\Gamma_{w}}{|\beta|}-\frac{\partial \Gamma_{w}}{\partial|\beta|} & =4 \frac{\Gamma_{w}}{|\beta|}=G_{1}^{\mathrm{Can}}-G_{2}^{\mathrm{Can}},
\end{aligned}
$$

where $\mathbb{A}^{\text {Can }}$ and $\mathbb{W}_{1,2}^{\text {Can }}$ are the thermal coefficients of Eq. (14) related to the mean value of canonical stress-energy tensor. Furthermore, because the axial current is dual to the spin tensor, we can show that

$$
\mathbb{S}=\frac{1}{2} n_{\mathrm{A}}, \quad \Gamma_{w}=\frac{1}{2} W^{\mathrm{A}} .
$$

Then, combining Eq.s (19) and (18), the coefficients of canonical stress-energy tensor and axial current are related by:

$$
\begin{aligned}
\mathbb{A}^{\mathrm{Can}} & =-\left(\frac{n_{\mathrm{A}}}{|\beta|}+\frac{\partial n_{\mathrm{A}}}{\partial|\beta|}\right), \\
\frac{n_{\mathrm{A}}}{|\beta|} & =\mathbb{W}_{1}^{\mathrm{Can}}-\mathbb{W}_{2}^{\mathrm{Can}}, \\
\frac{W^{\mathrm{A}}}{|\beta|} & =\frac{G_{1}^{\mathrm{Can}}-G_{2}^{\mathrm{Can}}}{2},
\end{aligned}
$$

which expose an interesting connection between the Axial Vortical Effect (AVE) conductivity $W^{\mathrm{A}}$ and the second order thermal coefficients of the canonical stressenergy tensor.

To understand the constraint (17) and the relation between axial vortical effect and anomalies, we also consider the case of a free massive field. In that case, axial current is not conserved, but its divergence is given by

$$
\partial_{\mu} \widehat{j}_{\mathrm{A}}^{\mu}=2 m i \bar{\Psi} \gamma^{5} \Psi
$$

It follows that global equilibrium with a conserved axial charge can not be reached. Then, to still use the previous global equilibrium analysis to massive field, we simply set the axial chemical potential to zero and we consider global equilibrium with just thermal vorticity and finite electric charge. As a consequence, the symmetries impose that all chiral coefficients (i.e. those which are not parity invariant) must 
be vanishing. However, the term in $W^{\mathrm{A}}$ of axial current decomposition is not chiral and consequently could be different from zero. Since the conservation equation is changed, we expect that also the condition (17) will be modified. We then have to consider the pseudo-scalar operator i $\mathrm{i} \bar{\Psi} \gamma^{5} \Psi$ that appears on the divergence of axial current. Pseudoscalar thermal expectation value can be decomposed at second order in thermal vorticity in the same way as other local operators and we find that it is given by a single term:

$$
\left\langle\mathrm{i} \bar{\Psi} \gamma^{5} \Psi\right\rangle=(\alpha \cdot w) L^{\alpha \cdot w}
$$

where the non chiral thermal coefficient can be obtained by

$$
L^{\alpha \cdot w}=\frac{1}{2}\left\langle\left\langle\left\{\widehat{K}_{3}, \widehat{J}_{3}\right\} i \bar{\Psi} \gamma^{5} \Psi\right\rangle\right\rangle .
$$

With this definition we find that the condition on axial vortical effect conductivity $W^{\mathrm{A}}$ becomes:

$$
|\beta| \frac{\partial W^{\mathrm{A}}}{\partial|\beta|}+3 W^{\mathrm{A}}=-2 m L^{\alpha \cdot w} .
$$

Differently from (17) the constraint (21) no longer imposes $W^{\mathrm{A}}$ to be proportional to the third power of temperature and $W^{\mathrm{A}}$ acquires terms which depends on the mass of the fields.

As concluding remarks we give some results for these coefficients for the free massless Dirac field. In that case this method reproduces the well-know [2] chiral vortical effect and axial vortical effect conductivities

$$
W^{\mathrm{V}}=\frac{\mu \mu_{\mathrm{A}} T}{\pi^{2}}, \quad W^{\mathrm{A}}=\frac{T^{3}}{6}+\frac{\left(\mu^{2}+\mu_{\mathrm{A}}^{2}\right) T}{2 \pi^{2}} .
$$

In the case of massive Dirac fields, global thermal equilibrium with thermal vorticity and vanishing axial chemical potential is well defined and the axial currents mean value can be directed along the rotation of the fluid. In that situation the AVE conductivity for a free massive Dirac field is [33]

$$
W^{A}=\frac{1}{2 \pi^{2}|\beta|} \int_{0}^{\infty} \mathrm{d} p\left[n_{F}\left(E_{p}-\mu\right)+n_{F}\left(E_{p}+\mu\right)\right] \frac{2 p^{2}+m^{2}}{E_{p}},
$$

where $E_{p}=\sqrt{p^{2}+m^{2}}$. This coefficient is related to pseudo-scalar thermal coefficient $L^{\alpha \cdot w}$ via Eq. (21) and indeed pseudo-scalar coefficient is given by

$$
L^{\alpha \cdot w}=-\frac{m}{4 \pi^{2} \beta^{2}} \int_{0}^{\infty} \frac{\mathrm{d} p}{E_{p}}\left[n_{F}^{\prime}\left(E_{p}-\mu\right)+n_{F}^{\prime}\left(E_{p}+\mu\right)\right],
$$

where the prime on distribution functions stands for derivative respect to $E_{p}$. We can give approximate results for integral in Eq. (23). For high temperature regime $(T \gg m)$, if the gas is non-degenerate $(|\mu|<m)$, we extract the AVE conductivity behavior using the Mellin transformation technique [35]. The result is 


$$
\frac{W^{\mathrm{A}}}{T} \simeq \frac{T^{2}}{6}+\frac{\mu^{2}}{2 \pi^{2}}-\frac{m^{2}}{4 \pi^{2}}-\frac{7 \zeta^{\prime}(-2) T^{2}}{8 \pi^{2}}\left(\frac{m}{T}\right)^{4}+O\left(\frac{m^{6}}{T^{6}}\right) .
$$

The first term in mass was also obtained in [36] where the axial vortical effect was evaluated with the statistical operator (6) but in curved space-time. Low temperature behavior can also be extracted from (23), see [33]. For a degenerate gas $(|\mu|>m)$ at zero temperature we obtain ${ }^{1}$ :

$$
\frac{W^{\mathrm{A}}}{T}=\frac{\mu^{2}}{2 \pi^{2}} \frac{\sqrt{\mu^{2}-m^{2}}}{\mu} .
$$

Instead for a non degenerate gas $(|\mu|<m)$ at low temperature $T<<m$ we have

$$
W^{\mathrm{A}} \approx\left(1+2 \frac{T}{m}\right) \frac{(m T)^{3 / 2}}{\sqrt{2} \pi^{3 / 2}} \mathrm{e}^{|\beta|(\mu-m)} .
$$

Axial current corrections for rotating and accelerating fluids is also discussed in [37, 38] for both massive and massless fields using an ansatz for Wigner function with thermal vorticity.

\section{Dirac Field in external electromagnetic field}

Consider a Dirac field in external electromagnetic field. The Lagrangian of the theory is given by

$$
\mathcal{L}=\frac{\mathrm{i}}{2}\left[\bar{\Psi} \gamma^{\mu} \vec{\partial}_{\mu} \Psi-\bar{\Psi} \gamma^{\mu} \overleftarrow{\partial}_{\mu} \Psi\right]-m \bar{\Psi} \Psi-\widehat{j}^{\mu} A_{\mu}
$$

where $\widehat{j}^{\mu}=q \bar{\Psi} \gamma^{\mu} \Psi, q$ is the elementary electric charge of the field and the gauge potential $A^{\mu}$ is an external non dynamic field. This Lagrangian is obtained from the free Dirac one with the minimal coupling substitution $\partial_{\mu} \rightarrow \partial_{\mu}+\mathrm{i} q A_{\mu}$ which ensures gauge invariance to the theory. From Euler Eq.s we obtain the equations of motion (EOM) for the Dirac field:

$$
\not \Psi=-\mathrm{i}(q \mathcal{A}+m) \Psi, \quad \not \bar{\Psi}=\bar{\Psi} \mathrm{i}(q \mathcal{A}+m) .
$$

By applying Noether's theorem to this Lagrangian we obtain the operators in Eq. (4).

\subsection{Symmetries in constant electromagnetic field}

It is worth noticing that the symmetries of the theory of fermions in external electromagnetic field are different from those of free fermions and from those of quantum

${ }^{1}$ Notice that $W^{\mathrm{A}} w^{\mu} \rightarrow\left(W^{\mathrm{A}} / T\right) \omega$, so there is no divergency for $T \rightarrow 0$. 
electrodynamics. While a system without external forces is symmetric for the full Poincaré group, some of the symmetries are lost when external fields are introduced. Indeed, external fields do not transform together with the rest of the system. In this section we discuss the symmetries of a system in the presence of an external constant homogeneous electromagnetic field. We will examine the transformations that are still symmetries of the theory, the consequent conserved quantities and the form of the generators of such transformations.

If the Lagrangian of our theory is invariant under translations, from Noether's theorem, we can identify four operators. Those operators share three properties: they are conserved quantities, they are the generators of translations and they constitute the four-momentum of the system. However, translation invariance by itself does not guarantees that the same quantity must have all the three above properties altogether. Consider again a system under an external electromagnetic field. In this situation, Poincaré symmetry of space-time is broken. Only in the special case of a constant and homogeneous electromagnetic field translation invariance is restored. However, the Lagrangian is not invariant under space-time translation, but it acquires a term that is a four-divergence. This term, under appropriate boundary conditions, does not affect the action of the system and the overall invariance is preserved. Nevertheless, the consequence of the additional term is that we can distinguish between three different operators, each of them having one of the three properties stated above. This is understood with the Noether-Tassie-Buchdahl theorem [39, 40, 41]: given a Lagrangian $\mathcal{L}\left(\Psi(x), \partial_{\mu} \Psi(x), x\right)$ and the infinitesimal transformation

$$
x^{\prime \mu}=x^{\mu}+\delta x^{\mu}, \quad \Psi^{\prime}=\Psi+\delta \Psi
$$

such that $\partial_{\mu} \delta x^{\mu}=0$, which transforms the Lagrangian in

$$
\mathcal{L}\left(\Psi^{\prime}\left(x^{\prime}\right), \partial_{\mu}^{\prime} \Psi^{\prime}\left(x^{\prime}\right), x^{\prime}\right)=\mathcal{L}\left(\Psi(x), \partial_{\mu} \Psi(x), x\right)+\partial_{\mu} X^{\mu},
$$

where $X^{\mu}$ is a functional depending exclusively on $\Psi(x)$ and $x$, the quantity

$$
\Gamma^{\mu}=\frac{\delta \mathcal{L}}{\delta \partial_{\mu} \Psi} \delta \Psi-\left(\frac{\delta \mathcal{L}}{\delta \partial_{\mu} \Psi} \partial_{\nu} \Psi-\mathcal{L} g_{v}^{\mu}\right) \delta x^{v}-X^{\mu}
$$

is conserved, i.e. divergence-less.

Consider the Dirac Lagrangian in constant homogeneous electromagnetic field

$$
\mathcal{L}\left(\Psi(x), \partial_{\mu} \Psi(x), x\right)=\bar{\Psi}(\mathrm{i} \not \partial-m) \Psi-\widehat{j}^{\mu} A_{\mu} .
$$

The translation transformation $\left(\delta \Psi=0, \delta x^{\mu}=\epsilon^{\mu}\right)$ acts on the Dirac field but does not act directly on the external gauge field. Therefore, a translation changes the Lagrangian by

$$
\begin{aligned}
\delta \mathcal{L} & =\mathcal{L}\left(\Psi^{\prime}\left(x^{\prime}\right), \partial_{\mu}^{\prime} \Psi^{\prime}\left(x^{\prime}\right), x^{\prime}\right)-\mathcal{L}\left(\Psi(x), \partial_{\mu} \Psi(x), x\right) \\
& =\widehat{j}^{\mu} \partial_{\nu} A_{\mu} \epsilon^{\nu}=\widehat{j}^{\mu}\left(F_{\nu \mu}+\partial_{\mu} A_{\nu}\right) \epsilon^{\nu}=-\widehat{j}^{\mu}\left(F_{\mu \nu}-\partial_{\mu} A_{\nu}\right) \epsilon^{\nu} .
\end{aligned}
$$


The quantity $X$ of Noether-Tassie-Buchdahl in this case is

$$
X^{\mu v} \equiv \widehat{j}^{\mu}\left(A^{v}+F^{v \lambda} x_{\lambda}\right) .
$$

Indeed its divergence is the variation of the Lagrangian

$\epsilon_{\nu} \partial_{\mu} X^{\mu \nu}=\left(\partial_{\mu} \widehat{j}^{\mu}\right)\left(A^{v}+F^{v \lambda} x_{\lambda}\right) \epsilon_{v}+\widehat{j}^{\mu}\left(\partial_{\mu} A_{v}+F_{\nu \mu}\right) \epsilon^{v}=-\widehat{j}^{\mu}\left(F_{\mu \nu}-\partial_{\mu} A_{\nu}\right) \epsilon^{\nu}=\delta \mathcal{L}$

Therefore the theorem implies that the system has a canonical conserved tensor given by:

$$
\widehat{\pi}_{\text {can }}^{\mu \nu}=\widehat{T}_{0}^{\mu v}-\widehat{j}^{\mu} A^{v}-\widehat{j}^{\mu} F^{v \lambda} x_{\lambda}
$$

where $\widehat{T}_{0}^{\mu \nu}$ is the free canonical Dirac stress-energy tensor. Using Belinfante procedure we can transform $\widehat{T}_{0}^{\mu \nu}-\widehat{j}^{\mu} A^{v}$ into the symmetric stress-energy tensor of Dirac field in external magnetic field $\widehat{T}_{S}^{\mu \nu}$ and the above conserved tensor can be written as:

$$
\widehat{\pi}^{\mu \nu} \equiv \widehat{T}_{S}^{\mu \nu}-\widehat{j}^{\mu} F^{v \lambda} x_{\lambda}
$$

From the above equation, we can simply verify that $\partial_{\mu} \widehat{\pi}^{\mu \nu}=0$ form Eq. (2). Note that $\widehat{\pi}^{\mu \nu}$ is not symmetric and that it is gauge invariant. The conserved quantities are obtained from the previous operators by

$$
\widehat{\pi}^{\mu}=\int \mathrm{d}^{3} x \widehat{\pi}^{0 \mu}
$$

and we can show that this four-vector constitutes the generators of the translation [41]. However, the momentum of the system is still given by

$$
\widehat{P}^{\mu}=\int \mathrm{d}^{3} x \widehat{T}^{0 \mu}
$$

but it is no longer a conserved quantity and it is no longer the generator of translations. Another difference with the four-momenta is that different components of this vectors do not commute, instead they satisfy the commutation relation [41]

$$
\left[\widehat{\pi}^{\mu}, \widehat{\pi}^{v}\right]=\mathrm{i} \widehat{Q} F^{\mu v}
$$

where $\widehat{Q}$ is the electric charge operator.

As for Lorentz's transformations we expect that the variation of the Lagrangian is a full divergence only for specific forms of transformations. For example, with a vanishing electric field and a constant magnetic field, only the rotation along the direction of the magnetic field and the boost along the magnetic field are symmetries of the theory. Therefore, only in these cases, the Lagrangian variation could be vanishing or a full divergence.

Returning to the general case, by repeating the previous argument made for the translations for the Lorentz transformation: 
Thermal chiral fermions with vorticity and magnetic field

$$
\delta x^{\mu}=\omega^{\mu \nu} x_{\nu}, \quad \delta \Psi=-\frac{\mathrm{i}}{2} \omega_{\mu \nu} \sigma^{\mu \nu} \Psi,
$$

we find that the transformed Lagrangian is

$$
\delta \mathcal{L}=\omega^{\mu v} \widehat{j}^{\lambda}\left[\partial_{\lambda}\left(x_{\mu} A_{v}-x_{\nu} A_{\mu}\right)+x_{\mu} F_{\nu \lambda}-x_{\nu} F_{\mu \lambda}\right] .
$$

We can show that the Lagrangian variation can also be written as

$$
\begin{aligned}
\delta \mathcal{L}= & \frac{1}{2} \omega^{\mu \nu} \partial_{\lambda}\left[\hat{j}^{\lambda} x_{\mu}\left(A_{\nu}-\frac{1}{2} x^{\sigma} F_{\sigma \nu}\right)-\widehat{j}^{\lambda} x_{\nu}\left(A_{\mu}-\frac{1}{2} x^{\sigma} F_{\sigma \mu}\right)\right] \\
& -\frac{1}{2} x^{\rho} \widehat{j}^{\lambda}\left(\omega_{\lambda \sigma} F_{\rho}^{\sigma}-\omega_{\rho \sigma} F_{\lambda}^{\sigma}\right) .
\end{aligned}
$$

The first term of the r.h.s. is written as a four-divergence. The remaining part can not be cast into a four-divergence but it is proportional to the following product

$$
(\omega \wedge F)_{\lambda \rho}=\omega_{\lambda \sigma} F_{\rho}^{\sigma}-\omega_{\rho \sigma} F_{\lambda}^{\sigma} .
$$

The product of two non-vanishing anti-symmetric tensor of rank two, $\omega \wedge F$, is zero if and only if $\omega$ is a linear combination of $F$ and its dual $F^{*}$ (or viceversa) [41]:

$$
(\omega \wedge F)_{\lambda \rho}=0 \quad \text { iff } \quad \omega_{\mu \nu}=k F_{\mu \nu}+k^{\prime} F_{\mu \nu}^{*}, \quad k, k^{\prime} \in \mathbb{R} .
$$

Therefore, the part of Lagrangian variation which is not a divergence is vanishing when $\omega^{\mu \nu}$ is a linear combination of electromagnetic stress-energy tensor and its dual:

$$
\omega^{\mu \nu}=a F^{\mu \nu}+\frac{b}{2} \epsilon^{\mu \nu \rho \sigma} F_{\rho \sigma} .
$$

This means, as expected, that the theory is invariant only under certain type of Lorentz transformations: the ones generated with parameters of the form (29). For example, in the case of constant magnetic field, we recover that the system is invariant only for rotation and boost along the magnetic field. Set then $\omega$ either as $\omega_{\mu \nu} \propto F_{\mu \nu}$ or $\omega_{\mu \nu} \propto F_{\mu \nu}^{*}$, so that the Lagrangian variation is a four-divergence. In this case we can apply Noether-Tassie-Buchdahl theorem and the two following quantities are divergence-less:

$$
\begin{aligned}
\widehat{\Gamma}^{\lambda}= & \frac{F^{\mu \nu}}{2}\left[x_{\mu}\left(\widehat{T}_{0 v}^{\lambda}-\widehat{j}^{\lambda} A_{\nu}+\frac{1}{2} \widehat{j}^{\lambda} x^{\rho} F_{\rho \nu}\right)\right. \\
& \left.-x_{\nu}\left(\widehat{T}_{0 \mu}^{\lambda}-\widehat{j}^{\lambda} A_{\mu}+\frac{1}{2} \widehat{j}^{\lambda} x^{\rho} F_{\rho \mu}\right)+\widehat{S}_{\mu \nu}^{\lambda}\right], \\
\widehat{\Gamma}^{* \lambda}= & \frac{F^{* \mu \nu}}{2}\left[x_{\mu}\left(\widehat{T}_{0 v}^{\lambda}-\widehat{j}^{\lambda} A_{\nu}+\frac{1}{2} \widehat{j}^{\lambda} x^{\rho} F_{\rho v}\right)\right. \\
& \left.-x_{\nu}\left(\widehat{T}_{0 \mu}^{\lambda}-\hat{j}^{\lambda} A_{\mu}+\frac{1}{2} \hat{j}^{\lambda} x^{\rho} F_{\rho \mu}\right)+\widehat{S}_{\mu \nu}^{\lambda}\right],
\end{aligned}
$$


where $\widehat{S}_{\mu \nu}^{\lambda}$ is the canonical spin tensor of free Dirac field. After Belinfante transformation, the quantities become

$$
\begin{aligned}
\widehat{\Gamma}^{\lambda} & =\frac{1}{2} F^{\mu \nu} \widehat{M}_{\mu \nu}^{\lambda}, \quad \widehat{\Gamma}^{* \lambda}=\frac{1}{2} F^{* \mu \nu} \widehat{M}_{\mu \nu}^{\lambda}, \\
\widehat{M}_{\mu \nu}^{\lambda} & \equiv x_{\mu}\left(\widehat{T}_{S \nu}^{\lambda}+\frac{1}{2} \widehat{j}^{\lambda} x^{\rho} F_{\rho \nu}\right)-x_{\nu}\left(\widehat{T}_{S \mu}^{\lambda}+\frac{1}{2} \widehat{j}^{\lambda} x^{\rho} F_{\rho \mu}\right) \\
& =x_{\mu}\left(\widehat{\pi}^{\lambda}{ }_{\nu}-\frac{1}{2} \widehat{j}^{\lambda} x^{\rho} F_{\rho \nu}\right)-x_{\nu}\left(\widehat{\pi}_{\mu}^{\lambda}-\frac{1}{2} \widehat{j}^{\lambda} x^{\rho} F_{\rho \mu}\right) .
\end{aligned}
$$

We can define the integrals:

$$
\widehat{M}_{\mu \nu}=\int \mathrm{d}^{3} x \widehat{M}_{\mu \nu}^{0},
$$

which are conserved quantities only if contracted with $F^{\mu \nu}$ or $F^{* \mu \nu}$. The operators $\widehat{M}_{\mu \nu}$ are the generators of Lorentz transformations if they are also a symmetry for the theory, otherwise the Wigner's theorem does not applies and we can not say that such transformations admit an unitary and linear (or anti-unitary and anti-linear) representation. For those operators, the following Algebra holds true [41]:

$$
\begin{aligned}
{\left[\widehat{\pi}^{\mu}, \widehat{\pi}^{\nu}\right] } & =\mathrm{i} F^{\mu \nu} \widehat{Q}, \\
\frac{1}{2} F_{\rho \sigma}\left[\widehat{\pi}^{\mu}, \widehat{M}^{\rho \sigma}\right] & =\frac{\mathrm{i}}{2} F_{\rho \sigma}\left(\eta^{\mu \rho} \widehat{\pi}^{\sigma}-\eta^{\mu \sigma} \widehat{\pi}^{\rho}\right), \\
\frac{1}{2} F_{\rho \sigma}^{*}\left[\widehat{\pi}^{\mu}, \widehat{M}^{\rho \sigma}\right] & =\frac{\mathrm{i}}{2} F_{\rho \sigma}^{*}\left(\eta^{\mu \rho} \widehat{\pi}^{\sigma}-\eta^{\mu \sigma} \widehat{\pi}^{\rho}\right),
\end{aligned}
$$

where $\widehat{Q}$ is the electric charge operator. In the particular case of vanishing electric field and constant magnetic field along the $z$ axis, the Algebra becomes:

$$
\begin{gathered}
{\left[\widehat{\pi}_{x}, \widehat{\pi}_{y}\right]=\mathrm{i}|\mathbf{B}| \widehat{Q},} \\
{\left[\widehat{J}_{z}, \widehat{\pi}_{x}\right]=\mathrm{i} \widehat{\pi}_{y}, \quad\left[\widehat{J}_{z}, \widehat{\pi}_{y}\right]=-\mathrm{i} \widehat{\pi}_{x},} \\
{\left[\widehat{K}_{z}, \widehat{\pi}_{t}\right]=-\mathrm{i} \widehat{\pi}_{z}, \quad\left[\widehat{K}_{z}, \widehat{\pi}_{z}\right]=-\mathrm{i} \widehat{\pi}_{t} .}
\end{gathered}
$$

\section{Chiral fermions in constant magnetic field}

Consider now a system consisting of free chiral fermions in an external homogeneous constant magnetic field $\mathbf{B}$ at global thermal equilibrium with vanishing thermal vorticity. In this configuration, the chiral anomaly is vanishing because there is no electric field $(B \cdot E=0)$. It follows that global equilibrium without vorticity is described by a constant inverse four-temperature $\beta$ and a constant axial chemical potential $\zeta_{\mathrm{A}}$ (see Sec. 2). Instead, the condition for the electric chemical potential reads 


$$
\partial^{\mu} \zeta(x)=F^{v \mu} \beta_{\nu}=\sqrt{\beta^{2}} F^{v \mu} u_{v}=\sqrt{\beta^{2}} E^{\mu},
$$

where $u$ is the fluid velocity directed along $\beta$ and $E$ is the comoving electric field. Since we are considering the case without electric field the global equilibrium condition is simply a constant $\zeta$. The global equilibrium statistical operator then becomes

$$
\widehat{\rho}=\frac{1}{Z} \exp \left[-\widehat{P}^{\mu} \beta_{\mu}+\zeta \widehat{Q}+\zeta_{\mathrm{A}} \widehat{Q}_{\mathrm{A}}\right] .
$$

Notice that the operators $\widehat{P}^{\mu}$ are not the generators of translations, which are instead given by $\widehat{\pi}^{\mu}$ and are obtained by integrating the conserved current in Eq. (27). However, in the case of vanishing comoving electric field, the projection of the inverse temperature along the four-momentum is equivalent to the projection along of the generators of translations, that is:

$$
\widehat{\pi}^{\mu} \beta_{\mu}=\int_{\Sigma} \mathrm{d} \Sigma_{\lambda}\left(\widehat{T}^{\lambda v}-\widehat{j}^{\lambda} F^{v \sigma} x_{\sigma}\right) \beta_{\nu}=\widehat{P}^{\mu} \beta_{\mu}-\sqrt{\beta^{2}} E^{\sigma} \int_{\Sigma} \mathrm{d} \Sigma_{\lambda} \widehat{j}^{\lambda} x_{\sigma}=\widehat{P}^{\mu} \beta_{\mu} .
$$

The statistical operator can now be written as

$$
\widehat{\rho}=\frac{1}{Z} \exp \left[-\widehat{\pi}^{\mu} \beta_{\mu}+\zeta \widehat{Q}+\zeta_{\mathrm{A}} \widehat{Q}_{\mathrm{A}}\right] .
$$

In this form, it is straightforward to use the algebra in Eq. (31) and translate the statistical operator of a quantity $a^{\mu}$. We find

$$
\widehat{\mathrm{T}}(a) \widehat{\rho} \widehat{\mathrm{T}}^{-1}(a)=\mathrm{e}^{\mathrm{i} a \cdot \widehat{\pi}} \widehat{\rho} \mathrm{e}^{-\mathrm{i} a \cdot \widehat{\pi}}=\frac{1}{Z} \exp \left[-\widehat{\pi}^{\mu} \beta_{\mu}+\zeta \widehat{Q}+\zeta_{\mathrm{A}} \widehat{Q}_{\mathrm{A}}+a_{\mu} F^{\mu v} \beta_{\nu} \widehat{Q}\right] .
$$

Since $F^{\mu \nu} \beta_{\nu}$ is the comoving electric field, which is vanishing, the statistical operator is homogeneous:

$$
\widehat{\mathrm{T}}(a) \widehat{\rho} \widehat{\mathrm{T}}^{-1}(a)=\widehat{\rho} .
$$

\subsection{Exact thermal solutions}

Having established the basic quantities of thermal equilibrium with a constant and homogeneous magnetic field, we now move on to introduce the techniques of thermal field theory in order to find exact solutions for thermodynamic equilibrium in a magnetic field. We start by giving a path integral description of the partition function. Since the partition function is a Lorentz invariant, we can choose the evaluate it in the local rest frame where $u=(1, \mathbf{0})$. In this frame, without loss in generality, the magnetic field is chosen along the $z$ axis and we adopt the Landau gauge $A^{\mu}=$ $\left(0,0, B x_{1}, 0\right)$. The path integral formulation of the partition function in local rest frame

$$
Z\left(T, \mu, \mu_{5}\right)=\operatorname{tr}\left[\mathrm{e}^{-\beta\left(\widehat{H}-\mu \widehat{Q}-\mu_{\mathrm{A}} \widehat{Q}_{\mathrm{A}}\right)}\right]
$$


is given by ${ }^{2}$

$$
Z=C \int_{\Psi(\beta, \mathbf{x})=-\Psi(0, \mathbf{x})} \mathcal{D} \bar{\Psi} \mathcal{D} \Psi \exp \left(-S_{\mathrm{E}}\left(\Psi, \bar{\Psi}, \mu_{\mathrm{A}}\right)\right)
$$

where the Euclidean action of Dirac fermions in external electromagnetic field is

$$
S_{\mathrm{E}}\left(\Psi, \bar{\Psi}, \mu_{5}\right)=\int_{0}^{\beta} \mathrm{d} \tau \int_{\mathbf{x}} \bar{\Psi}(X)\left[\mathrm{i}\left(\gamma \cdot \pi^{+}\right)+m-\gamma_{0} \gamma^{5} \mu_{\mathrm{A}}\right] \Psi(X)
$$

and $\pi_{\mu}^{+} \equiv P_{\mu}^{+}-q A_{\mu}$, which is not to be confused with the generators of translations.

With regard to the exact solution, instead of solving the Dirac equation directly, we use the Ritus method [42] (see [43] for a brief recap of the method). The core concept of Ritus method is that we can construct a complete set of orthonormal function, called $E_{p}$ Ritus functions, such that the Euclidean action is rendered formally identical to the Euclidean action of a free Dirac field in absence of external fields. The $E_{p}$ functions are constructed such that they are the matrix of the contemporaneous eigenfunctions (eigenvectors) of the maximal set of mutually commuting operators $\left\{(\gamma \cdot \pi)^{2}, \mathrm{i} \gamma_{1} \gamma_{2}, \gamma^{5}\right\}$. From gamma algebra, it is straightforward to check that

$$
\mathrm{i} \gamma_{1} \gamma_{2} \delta(\sigma)=\sigma \Delta(\sigma), \quad \frac{1+\chi \gamma^{5}}{2} \gamma^{5}=\chi \frac{1+\chi \gamma^{5}}{2},
$$

with $\sigma= \pm$ and $\chi= \pm$ and we defined

$$
\Delta(\sigma) \equiv \frac{1+\mathrm{i} \sigma \gamma_{1} \gamma_{2}}{2} .
$$

We can then show that $[42,43]$

$$
\left(\gamma \cdot \pi^{+}\right)^{2} E_{\widehat{p} \sigma}(X)=\underline{P}^{+2} E_{\widehat{p} \sigma}(X)
$$

where $\widehat{p}$ is a label for the quantum numbers $\left\{l, \omega_{n}, p_{2}, p_{3}\right\}$, the eigenvalues $\underline{P}^{+}$are given by

$$
\underline{P}^{+} \equiv\left(\omega_{n}+\mathrm{i} \mu, 0,-\bar{\sigma} \sqrt{2|q B| l}, p_{3}\right), \quad \bar{\sigma} \equiv \operatorname{sgn}(q B)
$$

and the form of eigenfunction is

$$
E_{\widehat{p} \sigma}(X)=N(n) \mathrm{e}^{\mathrm{i}\left(P_{0} \tau+P_{2} X_{2}+P_{3} X_{3}\right)} D_{n}(\rho)
$$

where $N(n)=(4 \pi|q B|)^{1 / 4} / \sqrt{n !}$ is a normalization factor, and $D_{n}(\rho)$ denotes the parabolic cylinder functions with argument $\rho=\sqrt{2|q B|}\left(X_{1}-p_{2} / q B\right)$ and nonnegative integer index $n=0,1,2, \ldots$ given by

$$
n=l+\frac{\sigma}{2} \operatorname{sgn}(q B)-\frac{1}{2}
$$

${ }^{2}$ We added a mass term for generalization, although with mass we cannot have a conserved axial current. 
Note that the form of the functions (32) strongly depends on the gauge chosen, in our case the Landau gauge. Since the eigenfunction $E_{\widehat{p} \sigma}(X)$ does not depend on chirality, the maximal eigenfunctions of the operators $\left\{(\gamma \cdot \pi)^{2}, \mathrm{i} \gamma_{1} \gamma_{2}, \gamma^{5}\right\}$ are given by

$$
E_{\widehat{p}}(X)=\sum_{\sigma= \pm}^{\prime} E_{\widehat{p} \sigma}(X) \Delta(\sigma), \quad \bar{E}_{\widehat{p}}(X)=\gamma_{0} E_{\widehat{p}}^{\dagger}(X) \gamma_{0}=\sum_{\sigma^{\prime}= \pm}^{\prime} E_{\widehat{p} \sigma^{\prime}}^{*}(X) \Delta\left(\sigma^{\prime}\right)
$$

where the prime on the summation symbol denotes that the sum is subject to the constraint

$$
\sigma=\left\{\begin{array}{ll}
\operatorname{sgn}(q B) & l=0 \\
\pm & l>0
\end{array} .\right.
$$

Some important properties can be derived from these definitions. Firstly, that the functions $E_{p}$ commute with $\gamma_{0}$ and with $\gamma^{5}$. Secondly, they satisfy the orthogonality relation

$$
\int_{X} \bar{E}_{\widehat{q}}(X) E_{\widehat{p}}(X)=(2 \pi)^{4} \widehat{\delta}^{(4)}(\widehat{p}-\widehat{q}) \Pi(l)
$$

where we defined

$$
\begin{aligned}
\delta^{(4)}\left(\widehat{p}-\widehat{p}^{\prime}\right) & \equiv \delta_{l, l^{\prime}} \beta \delta_{\omega_{n}, \omega_{n^{\prime}}} \delta\left(p_{2}-p_{2}^{\prime}\right) \delta\left(p_{3}-p_{3}^{\prime}\right) \\
\Pi(l) & \equiv\left\{\begin{array}{ll}
\frac{1+\mathrm{i} \bar{\sigma} \gamma_{1} \gamma_{2}}{2} & l=0 \\
1 & l>0
\end{array} .\right.
\end{aligned}
$$

And lastly, the action of the operator $\left(\gamma \cdot \pi^{+}\right)$on these function is

$$
\left(\gamma \cdot \pi^{+}\right) E_{\widehat{p}}(X)=E_{\widehat{p}}(X) \gamma \cdot \underline{P} .
$$

Since we showed that $E_{p}$ Ritus functions are a complete orthonormal functions, we can expand the Dirac fields in these functions:

$$
\begin{aligned}
& \Psi(X)=T \sum_{\left\{\omega_{n}\right\}} \sum_{l=0}^{\infty} \int \mathrm{d} p_{2} \int \frac{\mathrm{d} p_{3}}{(2 \pi)^{3}} E_{\widehat{p}}(X) \Psi(\underline{P}) \equiv \oiint_{\widehat{P}} E_{\widehat{p}}(X) \Psi(\underline{P}), \\
& \bar{\Psi}(X)=\oiint_{\widehat{Q}} \bar{\Psi}(\underline{Q}) \bar{E}_{\widehat{q}}(X) .
\end{aligned}
$$

Replacing this expansion on the Euclidean action, we find

$$
S_{\mathrm{E}}\left(\Psi, \bar{\Psi}, \mu_{5}\right)=\oiint_{\widehat{P}} \oiint_{\widehat{Q}} \int_{X} \bar{\Psi}(\underline{Q}) \bar{E}_{\widehat{q}}(X)\left[\mathrm{i}\left(\gamma \cdot \pi^{+}\right)+m-\gamma_{0} \gamma^{5} \mu_{\mathrm{A}}\right] E_{\widehat{p}}(X) \Psi(\underline{P})
$$

and, using the above mentioned properties of $E_{p}$ functions, we obtain

$$
S_{\mathrm{E}}\left(\Psi, \bar{\Psi}, \mu_{5}\right)=\oiint_{\widehat{P}} \bar{\Psi}(\underline{P}) \Pi(l)\left[\mathrm{i} \gamma \cdot \underline{P}^{+}+m-\gamma_{0} \gamma^{5} \mu_{\mathrm{A}}\right] \Psi(\underline{P}) .
$$


Notice that this is formally identical to the Euclidean action of free Dirac field.

We can now proceed to evaluate the partition function. We first change the integration variables in the partition function to the modes of $E_{p}$ functions $\bar{\Psi}(\underline{P})$ and $\Psi(\underline{P})$. The partition function is then a Gaussian integral of Grassmann variables, whose result is the exponent determinant. Hence the partition function becomes

$$
\begin{aligned}
Z= & \tilde{C} \int_{\Psi(\beta, \mathbf{x})=-\Psi(0, \mathbf{x})} \mathcal{D} \bar{\Psi}(\underline{P}) \mathcal{D} \Psi(\underline{P}) \times \\
& \times \exp \left\{-\oint_{\widehat{P}} \bar{\Psi}(\underline{P}) \Pi(l)\left[\mathrm{i} \gamma \cdot \underline{P}^{+}+m-\gamma_{0} \gamma^{5} \mu_{\mathrm{A}}\right] \Psi(\underline{P})\right\} \\
= & \tilde{C} \operatorname{det}\left[\Pi(l)\left(\mathrm{i} \gamma \cdot \underline{P}^{+}+m-\gamma_{0} \gamma^{5} \mu_{\mathrm{A}}\right)\right] .
\end{aligned}
$$

For the sake of clarity, for now on we will remove the factor $\Pi(l)$ :

$$
Z=\tilde{C} \operatorname{det}\left(\begin{array}{cc}
m \mathbb{I}_{2 \times 2} & {\left[\mathrm{i}\left(\omega_{n}+\mathrm{i} \mu\right)-\mu_{\mathrm{A}}\right] \mathbb{I}_{2 \times 2}+\sigma_{i} \underline{P}_{i}} \\
\left(\mathrm{i}\left(\omega_{n}+\mathrm{i} \mu\right)+\mu_{\mathrm{A}}\right) \mathbb{I}_{2 \times 2}-\sigma_{i} \underline{P}_{i} & m \mathbb{I}_{2 \times 2}
\end{array}\right) .
$$

The determinant is evaluated using the standard formula for block matrices

$$
\operatorname{det}\left(\begin{array}{ll}
A & B \\
C & D
\end{array}\right)=\operatorname{det}\left(A D-B D^{-1} C D\right)
$$

replacing that into the partition function we have

$$
\begin{aligned}
Z & =\tilde{C} \operatorname{det}\left[\left(\underline{P}^{+2}+m^{2}+\mu_{\mathrm{A}}^{2}\right) \mathbb{I}_{2 \times 2}-2 \sigma_{i} \underline{P}_{i} \mu_{\mathrm{A}}\right] \\
& =\tilde{C} \operatorname{det}\left[\left(\underline{P}^{+2}+m^{2}+\mu_{\mathrm{A}}^{2}\right)^{2}-4|\underline{\mathbf{P}}|^{2} \mu_{\mathrm{A}}^{2}\right] \\
& =\prod_{\omega_{n}, l, p_{3}} \tilde{C}\left[\left(\underline{P}^{+2}+m^{2}+\mu_{\mathrm{A}}^{2}\right)^{2}-4|\underline{\mathbf{P}}|^{2} \mu_{\mathrm{A}}^{2}\right],
\end{aligned}
$$

where we evaluated the determinant as the product of the eigenvalues of the matrix. To connect this quantity to the thermodynamics of the system, we are actually interested in its logarithm:

$$
\log Z=\sum_{\omega_{n}, l, p_{3}} \log \left[\left(\underline{P}^{+2}+m^{2}+\mu_{\mathrm{A}}^{2}\right)^{2}-4|\underline{\mathbf{P}}|^{2} \mu_{\mathrm{A}}^{2}\right]+\text { cnst. }
$$

In the next subsection we evaluate the thermodynamic potential of the system from the logarithm of partition function. Then, by simple derivation, we could obtain other thermodynamic properties. However, starting from partition function in Eq. (34), we will obtain the thermal propagator of a chiral fermion in a magnetic field. Once we have the propagator, we can use the point-splitting procedure to evaluate other thermal properties that are not related to the thermodynamic potential. We will use the thermal propagator to evaluate the mean value of the electric current and of the axial current in the following subsections. 


\subsection{Thermodynamic potential}

The thermodynamic potential $\Omega$ is derived from the partition function as following

$$
\Omega=\lim _{V \rightarrow \infty}-\frac{T}{V} \log Z
$$

where the logarithm of partition function is given by Eq. (35). We can follow the usual techniques used for free fermions to evaluate the thermodynamic potential and to sum the Matsubara frequencies. However, we must first consider that in this case the Landau levels generated by the magnetic field have different degeneracy factors ad must be properly taken into account when performing the infinite volume limit. Let be $S$ the area in the $x-y$ plane and $p_{\perp 1}$ and $p_{\perp 2}$ the momenta in that plane. In the limit of infinity area, the sum on modes becomes the following integrals:

$$
\lim _{S \rightarrow \infty} \frac{1}{S} \sum_{p_{\perp 1}} \sum_{p_{\perp 2}}=\int_{-\infty}^{\infty} \frac{\mathrm{d} p_{\perp 1}}{2 \pi} \int_{-\infty}^{\infty} \frac{\mathrm{d} p_{\perp 2}}{2 \pi} .
$$

Each Landau level has a degeneracy associated with some quantum numbers; this degeneracy is gauge independent and it is given by:

$$
d_{l}=\left\lfloor\frac{|q B| S}{2 \pi}\right\rfloor \text {. }
$$

To obtain this degeneracy, we just have to evaluate the quantity

$$
\frac{\mathrm{d} p_{\perp 1}}{2 \pi} \frac{\mathrm{d} p_{\perp 2}}{2 \pi}
$$

between two consecutive energy levels:

$$
d_{l}=\left\lfloor\frac{|q B| S}{2 \pi}\right\rfloor=\int_{l}^{l+1} \frac{\mathrm{d} p_{\perp 1}}{2 \pi} \frac{\mathrm{d} p_{\perp 2}}{2 \pi} .
$$

Therefore, removing the floor function, the infinite volume limit of the sum on the states of the system gives:

$$
\lim _{V \rightarrow \infty} \frac{1}{V} \sum_{\omega_{n}, l, p_{3}}=\frac{|q B|}{2 \pi} \sum_{l=0}^{\infty} \int_{-\infty}^{\infty} \frac{\mathrm{d} p_{3}}{2 \pi} \sum_{\left\{\omega_{n}\right\}} .
$$

Consequently the thermodynamic potential reads:

$$
\begin{aligned}
\Omega & =\lim _{V \rightarrow \infty}-\frac{T}{V} \log Z \\
& =-\frac{|q B|}{2 \pi} \sum_{l=0}^{\infty} \int_{-\infty}^{\infty} \frac{\mathrm{d} p_{3}}{2 \pi} T \sum_{\left\{\omega_{n}\right\}} \log \left[\left(\underline{P}^{+2}+m^{2}+\mu_{\mathrm{A}}^{2}\right)^{2}-4|\underline{\mathbf{P}}|^{2} \mu_{\mathrm{A}}^{2}\right]+\text { cnst. }
\end{aligned}
$$


The Matsubara sum can be performed as describe in [44]. The final result for thermodynamic potential is

$$
\Omega=-\frac{|q B|}{2 \pi} \sum_{l=0}^{\infty} \sum_{s= \pm}^{\prime} \int_{-\infty}^{\infty} \frac{\mathrm{d} p_{3}}{2 \pi}\left[E_{S}+T \sum_{ \pm} \log \left(1+\mathrm{e}^{-\beta\left(E_{s} \pm \mu\right)}\right)\right]+\mathrm{cnst},
$$

where $E_{s}^{2}=\left[\left(p_{3}^{2}+2 q B l\right)^{1 / 2}+s \mu_{\mathrm{A}}\right]^{2}+m^{2}$ and the constraint of $s=\bar{\sigma}$ for $l=0$ is caused by the projector $\Pi(l)$. This same thermodynamic potential for chiral fermions in external magnetic field was used in [4] to derive the chiral magnetic effect (CME). This expression can be used to obtain the electric and axial charge density, but instead we are using the point-splitting procedure because the latter can also be used to evaluate other thermodynamic functions related to currents. To do that, we first need the thermal propagator of chiral fermions.

\subsection{Chiral fermion propagator in magnetic field}

Since we have used the Ritus method, the form of Euclidean action is formally identical to those of a free Dirac field. It is therefore not surprising that the fermionic propagator is obtained in the same way as the free case. The propagator in Fourier modes can be obtained in path integral formulation by [44]

$$
\left\langle\tilde{\Psi}_{a}(P) \overline{\tilde{\Psi}}_{b}(Q)\right\rangle_{T}=\frac{\int \mathcal{D} \tilde{\Psi} \mathcal{D} \tilde{\bar{\Psi}} \exp \left(-S_{\mathrm{E}}\right) \tilde{\Psi}_{a}(P) \overline{\tilde{\Psi}}_{b}(Q)}{\int \mathcal{D} \tilde{\Psi} \mathcal{D} \tilde{\bar{\Psi}} \exp \left(-S_{\mathrm{E}}\right)},
$$

where in our case the form of the partition function is given in the first line of Eq. (34):

$$
\begin{aligned}
Z=\tilde{C} & \int_{\Psi(\beta, \mathbf{x})=-\Psi(0, \mathbf{x})} \mathcal{D} \bar{\Psi}(\underline{P}) \mathcal{D} \Psi(\underline{P}) \times \\
& \times \exp \left\{-\oint_{\widehat{P}} \bar{\Psi}(\underline{P}) \Pi(l)\left[\mathrm{i} \gamma \cdot \underline{P}^{+}+m-\gamma_{0} \gamma^{5} \mu_{\mathrm{A}}\right] \Psi(\underline{P})\right\} .
\end{aligned}
$$

The Grassmann integrals are straightforward and gives

$$
\left\langle\bar{\Psi}(\underline{Q})_{a} \Psi(\underline{P})_{b}\right\rangle=\delta^{(4)}(\underline{P}-\underline{Q}) \mathcal{M}_{a b}^{-1}
$$

where $a, b$ denotes spinorial indices and

$$
\mathcal{M}=\left[\mathrm{i} \gamma \cdot \underline{P}^{+}-\gamma_{0} \gamma^{5} \mu_{\mathrm{A}}\right] .
$$

The inverse of $\mathcal{M}$ is easily written in terms of the projector into right and left chirality states, which are defined by: 


$$
\mathbb{P}_{\chi}=\frac{1+\chi \gamma_{5}}{2}, \quad \text { i.e. } \quad \mathbb{P}_{\mathrm{R}}=\frac{1+\gamma_{5}}{2}, \quad \mathbb{P}_{\mathrm{L}}=\frac{1-\gamma_{5}}{2} .
$$

We also introduce right and left chemical potential:

$$
\mu_{\mathrm{R}} \equiv \mu+\mu_{\mathrm{A}}, \quad \mu_{\mathrm{L}} \equiv \mu-\mu_{\mathrm{A}},
$$

and we define right and left charged momenta by

$$
P_{\mathrm{R} / \mathrm{L}}^{ \pm} \equiv\left(\omega_{n} \pm \mathrm{i} \mu_{\mathrm{R} / \mathrm{L}}, \mathbf{p}\right) .
$$

With this notation, after inverting $\mathcal{M}$, the thermal propagator is

$$
\left\langle\bar{\Psi}(\underline{Q})_{a} \Psi(\underline{P})_{b}\right\rangle=\delta^{(4)}(\underline{P}-\underline{Q}) \sum_{\chi}\left(\mathbb{P}_{\chi} \frac{-\mathrm{i} \underline{P}_{\chi}^{+}}{\underline{P}_{\chi}^{+2}}\right)_{a b} .
$$

This is the generalization in Euclidean space-time with chemical potentials of the propagator in $[42,43]$. In the configuration space, the two-point function is

$$
\begin{aligned}
\left\langle\bar{\Psi}(X)_{a} \Psi(Y)_{b}\right\rangle & =\oiint_{\widehat{P}} \oiint_{\widehat{Q}} \bar{E}_{\widehat{p}}(X)_{a^{\prime} a} E_{\widehat{q}}(Y)_{b b^{\prime}}\left\langle\bar{\Psi}(\underline{P})_{a^{\prime}} \Psi(\underline{Q})_{b^{\prime}}\right\rangle \\
& =-\oiint_{\widehat{P}} \oiint_{\widehat{Q}} \bar{E}_{\widehat{p}}(X)_{a^{\prime} a} E_{\widehat{q}}(Y)_{b b^{\prime}}\left\langle\Psi(\underline{Q})_{b^{\prime}} \bar{\Psi}(\underline{P})_{a^{\prime}}\right\rangle \\
& =-\oiint_{\widehat{P}} \S_{\widehat{Q}} \bar{E}_{\widehat{p}}(X)_{a^{\prime} a} E_{\widehat{q}}(Y)_{b b^{\prime}} \delta^{(4)}(\underline{P}-\underline{Q}) \sum_{\chi}\left(\mathbb{P}_{\chi} \frac{-\mathrm{i} \underline{P}_{\chi}^{+}}{\underline{P}_{\chi}^{+2}}\right)_{b^{\prime} a^{\prime}}
\end{aligned}
$$

where to go to second line we used fermion anti-commutation. Finally, integrating the delta we have

$$
\left\langle\bar{\Psi}(X)_{a} \Psi(Y)_{b}\right\rangle=-\oiint_{\widehat{P}} \sum_{\chi} E_{\widehat{p}}(Y)_{b b^{\prime}}\left(\mathbb{P}_{\chi} \frac{-\mathrm{i} \underline{P}_{\chi}^{+}}{\underline{P}_{\chi}^{+2}}\right)_{b^{\prime} a^{\prime}} \bar{E}_{\widehat{p}}(X)_{a^{\prime} a}
$$

\subsection{Electric current mean value}

Having derived the propagator, we now proceed to evaluate the mean value of electric current. The following method is similar to the one used in [45], as we both use the Ritus method. We take advantage of the point-splitting procedure to compute the thermal expectation value of electric current. First, we write the current in Euclidean space-time and we split the coordinate point in which the fields are evaluated as follows:

$$
\left\langle\widehat{j}_{\mu}(X)\right\rangle=(-\mathrm{i})^{1-\delta_{\mu, 0}} q\left\langle\overline{\bar{\Psi}}(X) \gamma_{\mu} \widehat{\Psi}(X)\right\rangle=\lim _{X_{1}, X_{2} \rightarrow X}(-\mathrm{i})^{1-\delta_{\mu, 0}} q\left(\gamma_{\mu}\right)_{a b}\left\langle\widehat{\bar{\Psi}}_{a}\left(X_{1}\right) \widehat{\Psi}_{b}\left(X_{2}\right)\right\rangle
$$


Then we plug the form of fermionic propagator (36) and we reconstruct the trace on spinorial indices; eventually we obtain

$$
\left\langle\widehat{j}_{\mu}(X)\right\rangle=-(-\mathrm{i})^{1-\delta_{\mu, 0}} q \oiint_{\widehat{P}} \sum_{\chi} \operatorname{tr}\left[\bar{E}_{\widehat{p}}(X) \gamma_{\mu} E_{\widehat{p}}(X) \mathbb{P}_{\chi} \frac{-\mathrm{i} \underline{P}_{\chi}^{+}}{\underline{P}_{\chi}^{+2}}\right] .
$$

It is convenient to indicate the components parallel to the magnetic field, which are the time component and the $z$ component, with the parallel symbol "\|l". For those components the following commutator holds true:

$$
\left[\gamma_{\mu}^{\|}, E_{\widehat{p}}(X)\right]=0
$$

Therefore, reminding the definitions of the Ritus $E_{\widehat{p}}(X)$ functions (33), for the parallel component of electric current we obtain

$$
\begin{aligned}
\left\langle\hat{j}_{\mu}^{\|}(X)\right\rangle= & -(-\mathrm{i})^{1-\delta_{\mu, 0}} q \sum_{l=0}^{\infty} \int \frac{\mathrm{d} p_{2}}{2 \pi} \int \frac{\mathrm{d} p_{3}}{(2 \pi)^{2}} \sum_{\chi} T \sum_{\left\{\omega_{n}\right\}} \sum_{\sigma, \sigma^{\prime}= \pm}^{\prime} E_{\widehat{p} \sigma^{\prime}}^{*}(X) E_{\widehat{p} \sigma}(X) \times \\
& \times \operatorname{tr}\left[\Delta\left(\sigma^{\prime}\right) \Delta(\sigma) \gamma_{\mu}^{\|} \mathbb{P}_{\chi} \frac{-\mathrm{i} \mathbb{P}_{\chi}^{+}}{\underline{P}_{\chi}^{+2}}\right] .
\end{aligned}
$$

We can simplify the previous expression by taking advantage of the following identity

$$
\Delta(\sigma) \Delta\left(\sigma^{\prime}\right)=\frac{1+\sigma \sigma^{\prime}+\mathrm{i}\left(\sigma+\sigma^{\prime}\right) \gamma_{1} \gamma_{2}}{4}=\delta_{\sigma, \sigma^{\prime}} \Delta(\sigma) .
$$

Notice that the dependence on $p_{2}$ is only contained inside $E_{\widehat{p} \sigma^{\prime}}^{*}(X) E_{\widehat{p} \sigma}(X)$. We can then show that the integration on $p_{2}$ gives

$$
\int_{-\infty}^{\infty} \frac{\mathrm{d} p_{2}}{2 \pi} E_{\widehat{p} \sigma^{\prime}}^{*}(X) E_{\widehat{p} \sigma}(X)=|q B| \delta_{n, n^{\prime}}
$$

Furthermore, it is convenient to split the sum on $l$ between the lowest Landau level (LLL) $l=0$ and the higher Landau levels (HLL) $l>1$. For $l=0$ the sums on $\sigma$ are constrained to be equal to $\sigma=\sigma^{\prime}=\bar{\sigma}=\operatorname{sgn}(e B)$, and the momenta are given by $\underline{P}_{\chi}^{+}=\left(\omega_{n}+\mathrm{i} \mu_{\chi}, 0,0, p_{3}\right)$; then at the lowest Landau level we have

$$
\left\langle\hat{j}_{\mu}^{\|}(X)\right\rangle_{\mathrm{LLL}}=-(-\mathrm{i})^{1-\delta_{\mu, 0}} q|q B| \int_{-\infty}^{\infty} \frac{\mathrm{d} p_{3}}{(2 \pi)^{2}} \sum_{\chi} T \sum_{\left\{\omega_{n}\right\}} \operatorname{tr}\left[\frac{1+\mathrm{i} \bar{\sigma} \gamma_{1} \gamma_{2}}{2} \gamma_{\mu}^{\|} \mathbb{P}_{\chi} \frac{-\mathrm{i} \underline{P}_{\chi}^{+}}{\underline{P}_{\chi}^{+2}}\right] .
$$

After computing the trace, we find that the zero component is 
Thermal chiral fermions with vorticity and magnetic field

$$
\begin{aligned}
\left\langle\widehat{j_{0}}(X)\right\rangle_{\text {LLL }} & =-\int_{-\infty}^{\infty} \frac{q|q B| \mathrm{d} p_{3}}{(2 \pi)^{2}} \sum_{\chi} T \sum_{\left\{\omega_{n}\right\}} \frac{-\mathrm{i}\left[\left(\omega_{n}+\mathrm{i} \mu_{\chi}\right)-\mathrm{i} p_{3} \bar{\sigma} \chi\right]}{\left(\omega_{n}+\mathrm{i} \mu_{\chi}\right)^{2}+p_{3}^{2}} \\
& =\int_{-\infty}^{\infty} \frac{q|q B| \mathrm{d} p_{3}}{(2 \pi)^{2}} \sum_{\chi} T \sum_{\left\{\omega_{n}\right\}} \frac{\mathrm{i}\left[\left(\omega_{n}+\mathrm{i} \mu_{\chi}\right)\right]}{\left(\omega_{n}+\mathrm{i} \mu_{\chi}\right)^{2}+p_{3}^{2}}
\end{aligned}
$$

while the $z$ component is

$$
\begin{aligned}
\left\langle\widehat{j_{3}}(X)\right\rangle_{\text {LLL }} & =\mathrm{i} \int_{-\infty}^{\infty} \frac{q|q B| \mathrm{d} p_{3}}{(2 \pi)^{2}} \sum_{\chi} T \sum_{\left\{\omega_{n}\right\}} \frac{-\mathrm{i}\left[p_{3}+\mathrm{i}\left(\omega_{n}+\mathrm{i} \mu_{\chi}\right) \bar{\sigma} \chi\right]}{\left(\omega_{n}+\mathrm{i} \mu_{\chi}\right)^{2}+p_{3}^{2}} \\
& =\int_{-\infty}^{\infty} \frac{\bar{\sigma} q|q B| \mathrm{d} p_{3}}{(2 \pi)^{2}} \sum_{\chi} T \sum_{\left\{\omega_{n}\right\}} \frac{\chi \mathrm{i}\left(\omega_{n}+\mathrm{i} \mu_{\chi}\right)}{\left(\omega_{n}+\mathrm{i} \mu_{\chi}\right)^{2}+p_{3}^{2}}
\end{aligned}
$$

where the linear terms in $p_{3}$ were dropped because they are odd on $p_{3}$ and as such they vanish when integrated. After the Matsubara sum we have

$$
\begin{aligned}
\left\langle\widehat{j_{0}}(X)\right\rangle_{\mathrm{LLL}} & =q|q B| \sum_{\chi} \int_{-\infty}^{\infty} \frac{\mathrm{d} p_{3}}{(2 \pi)^{2}} \frac{1}{2}\left[n_{\mathrm{F}}\left(p_{3}-\mu_{\chi}\right)-n_{\mathrm{F}}\left(p_{3}+\mu_{\chi}\right)\right], \\
\left\langle\widehat{j_{3}}(X)\right\rangle_{\mathrm{LLL}} & =q^{2} B \sum_{\chi} \int_{-\infty}^{\infty} \frac{\mathrm{d} p_{3}}{(2 \pi)^{2}} \frac{\chi}{2}\left[n_{\mathrm{F}}\left(p_{3}-\mu_{\chi}\right)-n_{\mathrm{F}}\left(p_{3}+\mu_{\chi}\right)\right] .
\end{aligned}
$$

Finally, taking advantage of the integral in $p_{3}$ :

$$
\int_{-\infty}^{\infty} \frac{\mathrm{d} p_{3}}{2}\left[n_{\mathrm{F}}\left(p_{3}-\mu_{\chi}\right)-n_{\mathrm{F}}\left(p_{3}+\mu_{\chi}\right)\right]=\mu_{\chi}
$$

and summing on chiralities, we obtain

$$
\begin{aligned}
& \left\langle\widehat{j_{0}}(X)\right\rangle_{\mathrm{LLL}}=\frac{q|q B|}{(2 \pi)^{2}}\left(\mu_{\mathrm{R}}+\mu_{\mathrm{L}}\right)=\frac{\mu q|q B|}{2 \pi^{2}}, \\
& \left\langle\widehat{j_{3}}(X)\right\rangle_{\mathrm{LLL}}=\frac{q^{2} B}{(2 \pi)^{2}}\left(\mu_{\mathrm{R}}-\mu_{\mathrm{L}}\right)=\frac{q^{2} \mu_{\mathrm{A}}}{2 \pi^{2}} B .
\end{aligned}
$$

Moving on now to the higher Landau levels, consider

$$
\begin{aligned}
\left\langle\widehat{j}_{\mu}^{\|}(X)\right\rangle_{\mathrm{HLL}}= & -(-\mathrm{i})^{1-\delta_{\mu, 0}} q|q B| \sum_{l=1}^{\infty} \int_{-\infty}^{\infty} \frac{\mathrm{d} p_{3}}{(2 \pi)^{2}} \sum_{\chi} T \sum_{\left\{\omega_{n}\right\}} \sum_{\sigma, \sigma^{\prime}= \pm} \delta_{n, n^{\prime}} \times \\
& \times \operatorname{tr}\left[\Delta\left(\sigma^{\prime}\right) \Delta(\sigma) \gamma_{\mu}^{\|} \mathbb{P}_{\chi} \frac{-\mathrm{i} \mathbb{P}_{\chi}^{+}}{\underline{P}_{\chi}^{+2}}\right] .
\end{aligned}
$$

When $l$ is fixed we can replace the $\delta_{n, n^{\prime}}$ with the $\delta_{\sigma, \sigma^{\prime}}$ and the sum on $\sigma^{\prime}$ becomes straightforward. The expression is similar to the LLL case, we just have to replace $\bar{\sigma}$ with $\sigma$ and sum over $\sigma= \pm$. Remind that now $\underline{P}$ has also a $y$ component. After 
evaluating the trace and removing $p_{3}$ odd terms, we obtain

$$
\begin{aligned}
& \left\langle\widehat{j_{0}}(X)\right\rangle_{\mathrm{HLL}}=\sum_{l=1}^{\infty} \int_{-\infty}^{\infty} \frac{q|q B| \mathrm{d} p_{3}}{(2 \pi)^{2}} \sum_{\chi} T \sum_{\left\{\omega_{n}\right\}} \frac{2 \mathrm{i}\left[\left(\omega_{n}+\mathrm{i} \mu_{\chi}\right)\right]}{P_{\chi}^{+2}}, \\
& \left\langle\widehat{j}_{3}(X)\right\rangle_{\mathrm{HLL}}=\sum_{l=1}^{\infty} \int_{-\infty}^{\infty} \frac{q|q B| \mathrm{d} p_{3}}{(2 \pi)^{2}} \sum_{\chi} T \sum_{\left\{\omega_{n}\right\}} \sum_{\sigma= \pm} \sigma \frac{\chi \mathrm{i}\left(\omega_{n}+\mathrm{i} \mu_{\chi}\right)+p_{3}}{\underline{P}_{\chi}^{+2}}=0 .
\end{aligned}
$$

We found that the third component does not get corrections from HLL. Instead for the time component, after the frequency sum, we obtain

$$
\left\langle\widehat{j_{0}}(X)\right\rangle_{\mathrm{HLL}}=\sum_{l=1}^{\infty} \int_{-\infty}^{\infty} \frac{q|q B| \mathrm{d} p_{3}}{(2 \pi)^{2}} \sum_{\chi}\left[n_{\mathrm{F}}\left(E_{p_{3}, l}-\mu_{\chi}\right)-n_{\mathrm{F}}\left(E_{p_{3}, l}+\mu_{\chi}\right)\right],
$$

where $E_{p_{3}, l} \equiv \sqrt{p_{3}^{2}+2|q B| l}$. For the perpendicular components $\left(\widehat{j_{x}}\right.$ and $\widehat{j_{y}}$ ) we expect a vanishing result because they are not allowed by the symmetries of the system. Indeed the explicit calculations confirmed this expectation.

In summary, restoring covariant expression, we found that the electric current has two thermodynamic function: the electric charge density $n_{\mathrm{c}}$ and the chiral magnetic effect (CME) conductivity $\sigma_{\mathrm{B}}$ :

$$
\left\langle\widehat{j_{\mu}}(X)\right\rangle=n_{\mathrm{c}} u_{\mu}+\sigma_{\mathrm{B}} B_{\mu} .
$$

The electric charge density is given by the mean value $\left\langle\widehat{j_{0}}(X)\right\rangle$ at the local rest frame:

$$
\begin{aligned}
n_{\mathrm{c}}=\frac{q|q B|}{2 \pi^{2}}\left\{\mu+\sum_{l=1}^{\infty} \int_{-\infty}^{\infty} \frac{\mathrm{d} p_{3}}{2}\right. & {\left[n_{\mathrm{F}}\left(E_{p_{3}, l}-\mu_{\mathrm{R}}\right)-n_{\mathrm{F}}\left(E_{p_{3}, l}+\mu_{\mathrm{R}}\right)+\right.} \\
& \left.\left.+n_{\mathrm{F}}\left(E_{p_{3}, l}-\mu_{\mathrm{L}}\right)-n_{\mathrm{F}}\left(E_{p_{3}, l}+\mu_{\mathrm{L}}\right)\right]\right\},
\end{aligned}
$$

while the CME conductivity is given by $\left\langle\widehat{j}_{3}(X)\right\rangle / B$, that is

$$
\sigma_{\mathrm{B}}=\frac{q^{2} \mu_{\mathrm{A}}}{2 \pi^{2}} .
$$

To our knowledge the equation for the electric charge density of an electron gas in a magnetic medium was first given in [8] and coincides with the expression above. The CME effect evaluated here coincides with the one obtained with many other derivations [2], however we want to point out that this derivation is valid at thermal equilibrium, as the one in [46], and that is non-perturbative in magnetic field. 


\subsection{Axial current mean value}

We can compute the axial current mean value exactly as described above for the electric current. Because of that we omit all the calculations. The axial current constitutive equation is written in terms of an axial charge density $n_{\mathrm{A}}$ and a Chiral Separation Effect (CSE) conductivity $\sigma_{\mathrm{s}}$ :

$$
\left\langle\widehat{j}_{A \mu}\right\rangle=n_{\mathrm{A}} u_{\mu}+\sigma_{\mathrm{s}} B_{\mu} .
$$

In this case too we found that only the lowest Landau level contributes to CSE and that the final result is

$$
\begin{aligned}
n_{\mathrm{A}}= & \frac{|q B|}{2 \pi^{2}}\left\{\mu_{\mathrm{A}}+\sum_{l=1}^{\infty} \int_{-\infty}^{\infty} \frac{\mathrm{d} p_{3}}{2}\left[n_{\mathrm{F}}\left(E_{p_{3}, l}-\mu_{\mathrm{R}}\right)-n_{\mathrm{F}}\left(E_{p_{3}, l}+\mu_{\mathrm{R}}\right)+\right.\right. \\
& \left.\left.-n_{\mathrm{F}}\left(E_{p_{3}, l}-\mu_{\mathrm{L}}\right)+n_{\mathrm{F}}\left(E_{p_{3}, l}+\mu_{\mathrm{L}}\right)\right]\right\}, \\
\sigma_{\mathrm{S}}= & \frac{q \mu}{2 \pi^{2}},
\end{aligned}
$$

with $E_{p_{3}, l}=\sqrt{p_{3}^{2}+2|q B| l}$. The last thermal coefficient is exactly the well known value of Chiral Separation Effect (CSE) conductivity [2].

The same procedure can be followed to evaluate the axial charge density and the CSE conductivity of massive fermions with vanishing axial chemical potential $\mu_{\mathrm{A}}=0$. In that case, as discussed previously, the thermal equilibrium can be reached and all the quantity discussed in this section are still well defined. The results for the thermodynamic functions related to axial current are:

$$
\begin{aligned}
& n_{\mathrm{A}}=0, \\
& \sigma_{\mathrm{s}}=q B \int_{-\infty}^{\infty} \frac{\mathrm{d} p_{3}}{(2 \pi)^{2}}\left[n_{\mathrm{F}}\left(E_{p_{3}}-\mu\right)-n_{\mathrm{F}}\left(E_{p_{3}}+\mu\right)\right],
\end{aligned}
$$

with $E_{p_{3}}^{2}=p_{3}^{2}+m^{2}$. The CSE induces an axial current even if the system is not chiral. It is apparent from the result above that CSE has an explicit mass dependence, as it is known that it should have [47]. 


\section{Constant vorticity and electromagnetic field}

So far this contribution has focused on the general properties of the statistical operator of global thermodynamic equilibrium with both vorticity and electromagnetic field (12). The following section will discuss the special case of a constant homogeneous electromagnetic field ( $F^{\mu \nu}=$ constant) for which we already studied the symmetries (Sec. 3.1). As discussed in Sec. 2, global equilibrium can only be reached if condition (9) is satisfied, which in this case becomes

$$
\mathcal{L}_{\beta}\left(F^{\mu \nu}\right)=\varpi_{\sigma}^{\mu} F^{\sigma v}-\varpi^{v}{ }_{\sigma} F^{\sigma \mu} \equiv(\varpi \wedge F)^{\mu \nu}=0 .
$$

We already discussed this wedge product in Eq. (28). The Eq. (39) has two independent solutions: $F=k \varpi$ and $F=k^{\prime} \varpi^{*}$, with $k$ and $k^{\prime}$ real numbers. In terms of the gauge potential the condition (10) must be satisfied. From Eq. (39), choosing the covariant gauge $A^{\mu}=\frac{1}{2} F^{\rho \mu} x_{\rho}$, we find that condition (10) is satisfied setting $\Phi=\frac{1}{2} b_{\sigma} F^{\sigma \lambda} x_{\lambda}$. The equilibrium chemical potential (11) is then written as

$$
\zeta(x)=\zeta_{0}-\beta_{\sigma}(x) F^{\lambda \sigma} x_{\lambda}+\frac{1}{2} \varpi_{\sigma \rho} x^{\rho} F^{\lambda \sigma} x_{\lambda} .
$$

The same solution can also be obtained by directly solving Eq. (7) using Eq. (39). This last method to obtain the solution explicitly shows that the chemical potential in Eq. (40) is not gauge dependent. For constant magnetic field and vanishing thermal vorticity the solution (40) reduces to $\zeta=$ constant, as it was correctly used in Sec. 4.

Plugging the form (40) inside the operator of Eq. (1) we find:

$$
\widehat{\rho}=\frac{1}{Z} \exp \left\{-\int \mathrm{d} \Sigma_{\lambda}\left[\left(\widehat{T}^{\lambda v}-\widehat{j}^{\lambda} F^{v \rho} x_{\rho}\right) \beta_{v}-\frac{1}{2} \varpi_{\sigma \rho} \hat{j}^{\lambda} x^{\rho} F^{\tau \sigma} x_{\tau}-\zeta_{0} \hat{j}^{\lambda}\right]\right\} .
$$

Inside the round bracket we recognize the divergence-less operator $\widehat{\pi}^{\lambda v}$ of Eq. (27), whose integrals are the generators of translations. Expressing the coordinate dependence of $\beta$, we can then write

$$
\begin{aligned}
& \widehat{\rho}=\frac{1}{Z} \exp \left\{-\int \mathrm{d} \Sigma_{\lambda}\left[\widehat{\pi}^{\lambda v} b_{v}+\varpi_{v \tau} x^{\tau} \widehat{\pi}^{\lambda v}-\frac{1}{2} \varpi_{\mu \nu} \hat{j}^{\lambda} x^{v} F^{\tau \mu} x_{\tau}-\zeta_{0} \hat{j}^{\lambda}\right]\right\} \\
& =\frac{1}{Z} \exp \left\{-\int \mathrm{d} \Sigma_{\lambda}\left[\widehat{\pi}^{\lambda v} b_{v}+\varpi_{\mu \nu} x^{v}\left(\widehat{\pi}^{\lambda \mu}-\frac{1}{2} \widehat{j}^{\lambda} F^{\rho \mu} x_{\rho}\right)-\zeta_{0} \widehat{j}^{\lambda}\right]\right\} \\
& =\frac{1}{Z} \exp \left\{-\int \mathrm{d} \Sigma_{\lambda}\left[\widehat{\pi}^{\lambda v} b_{v}-\frac{1}{2} \varpi_{\mu \nu}\left[x^{\mu}\left(\widehat{\pi}^{\lambda v}-\frac{1}{2} \widehat{j}^{\lambda} F^{\rho v} x_{\rho}\right)\right.\right.\right. \\
& \left.\left.\left.-x^{\nu}\left(\widehat{\pi}^{\lambda \mu}-\frac{1}{2} \hat{j}^{\lambda} F^{\rho \mu} x_{\rho}\right)\right]-\zeta_{0} \hat{j}^{\lambda}\right]\right\}
\end{aligned}
$$

this time we have recreated the divergence-less quantity $\varpi_{\mu v} \widehat{M}^{\lambda, \mu v}$ of Eq. (30) that generates the Lorentz transformations and that are symmetries of the system. We can then integrate over the coordinate and we find: 


$$
\widehat{\rho}=\frac{1}{Z} \exp \left\{-b \cdot \widehat{\pi}+\frac{1}{2} \varpi: \widehat{M}+\zeta_{0} \widehat{Q}\right\} .
$$

In the above form, the analogy with statistical operator without electromagnetic field in Eq. (6) is evident. In both cases the statistical operator is written with the sum of conserved operators, each one weighted with a constant Lagrange multiplier. Moreover, starting from a fixed point $x$ we can write the constants thermal fields as

$$
b_{\mu}=\beta(x)_{\mu}-\varpi_{\mu \nu} x^{\nu}, \quad \zeta_{0}=\zeta(x)+\beta_{\sigma}(x) F^{\lambda \sigma} x_{\lambda}-\frac{1}{2} \varpi_{\sigma \rho} x^{\rho} F^{\lambda \sigma} x_{\lambda},
$$

from which the statistical operator becomes

$$
\begin{aligned}
\widehat{\rho}= & \frac{1}{Z} \exp \left\{-\beta(x)_{\mu}\left(\widehat{\pi}^{\mu}-F^{\lambda \mu} x_{\lambda} \widehat{Q}\right)+\right. \\
& \left.+\frac{1}{2} \varpi_{\mu \nu}\left(\widehat{M}^{\mu \nu}+x^{v} \widehat{\pi}^{\mu}-x^{\mu} \widehat{\pi}^{v}-x^{\nu} F^{\lambda \mu} x_{\lambda} \widehat{Q}\right)+\zeta(x) \widehat{Q}\right\} .
\end{aligned}
$$

It is important to point out that with an external magnetic field the Poincare algebra is modified and becomes the Algebra in Eq. (31), which we report here for convenience:

$$
\begin{aligned}
{\left[\widehat{\pi}^{\mu}, \widehat{\pi}^{v}\right] } & =\mathrm{i} F^{\mu \nu} \widehat{Q}, \\
\frac{1}{2} F_{\rho \sigma}\left[\widehat{\pi}^{\mu}, \widehat{M}^{\rho \sigma}\right] & =\frac{\mathrm{i}}{2} F_{\rho \sigma}\left(\eta^{\mu \rho} \widehat{\pi}^{\sigma}-\eta^{\mu \sigma} \widehat{\pi}^{\rho}\right), \\
\frac{1}{2} F_{\rho \sigma}^{*}\left[\widehat{\pi}^{\mu}, \widehat{M}^{\rho \sigma}\right] & =\frac{\mathrm{i}}{2} F_{\rho \sigma}^{*}\left(\eta^{\mu \rho} \widehat{\pi}^{\sigma}-\eta^{\mu \sigma} \widehat{\pi}^{\rho}\right) .
\end{aligned}
$$

Notice that because $F$ is proportional to $\varpi$, if we replace $F$ with $\varpi$ and $F^{*}$ with $\varpi^{*}$ the last two algebra identities still hold true. Since the Algebra is known, we can translate the statistical operator. Taking advantage of the unitary of the translation transformation, the translated statistical operator is

$$
\begin{aligned}
\widehat{\mathrm{T}}(x) \widehat{\rho} \widehat{\mathrm{T}}^{-1}(x)= & \frac{1}{Z} \exp \left\{-\widehat{\mathrm{T}}(x)(b \cdot \widehat{\pi}) \widehat{\mathrm{T}}^{-1}(x)+\right. \\
& \left.+\widehat{\mathrm{T}}(x)\left(\frac{\varpi: \widehat{M}}{2}\right) \widehat{\mathrm{T}}^{-1}(x)+\zeta_{0} \widehat{\mathrm{T}}(x) \widehat{Q} \widehat{\mathrm{T}}^{-1}(x)\right\} .
\end{aligned}
$$

Therefore we just need to evaluate how the operators $\widehat{\pi}, \widehat{M}$ and $\widehat{Q}$ transform under translations. For a unitary transformation an operator $\widehat{K}$ transforms with

$$
\mathrm{e}^{\mathrm{i} \widehat{A}} \widehat{K} \mathrm{e}^{-\mathrm{i} \widehat{A}} \simeq \widehat{K}-\mathrm{i}[\widehat{K}, \widehat{A}]-\frac{1}{2}[[\widehat{K}, \widehat{A}], \widehat{A}]+\frac{\mathrm{i}}{6}[[[\widehat{K}, \widehat{A}], \widehat{A}], \widehat{A}]+\cdots
$$

By applying this formula to our operators we obtain the complete transformation because after a certain order all the commutators become vanishing. In particular, for the Lorentz transformation generators we find 
$\frac{1}{2} \varpi_{\mu \nu} \widehat{M}_{x}^{\mu \nu} \equiv \frac{1}{2} \varpi_{\mu \nu} \widehat{\top}(x) \widehat{M}^{\mu \nu} \widehat{\top}^{-1}(x)=\frac{1}{2} \varpi_{\mu \nu}\left(\widehat{M}^{\mu \nu}+x^{\nu} \widehat{\pi}^{\mu}-x^{\mu} \widehat{\pi}^{\nu}-x^{\nu} F^{\lambda \mu} x_{\lambda} \widehat{Q}\right)$.

For the other operators instead we find:

$$
\widehat{\pi}_{x}^{\mu} \equiv \widehat{\mathrm{T}}(x) \widehat{\pi}^{\mu} \widehat{\mathrm{T}}^{-1}(x)=\widehat{\pi}^{\mu}-x_{\rho} F^{\rho \mu} \widehat{Q}, \quad \widehat{\mathrm{T}}(x) \widehat{Q} \widehat{\mathrm{T}}^{-1}(x)=\widehat{Q} .
$$

With these definitions a translation transformation on the statistical operator act as following:

$$
\begin{aligned}
\widehat{\mathrm{T}}(a) \widehat{\rho} \widehat{\mathrm{T}}^{-1}(a) & =\frac{1}{Z} \exp \left\{-\beta(x) \cdot \widehat{\pi}_{x+a}+\frac{1}{2} \varpi: \widehat{M}_{x+a}+\zeta(x) \widehat{Q}\right\} \\
& =\frac{1}{Z} \exp \left\{-\beta(x-a) \cdot \widehat{\pi}_{x}+\frac{1}{2} \varpi: \widehat{M}_{x}+\zeta(x-a) \widehat{Q}\right\} .
\end{aligned}
$$

It follows that the statistical operator around a point $x$ can be written as:

$$
\widehat{\rho}=\frac{1}{Z} \exp \left\{-\beta(x) \cdot \widehat{\pi}_{x}+\frac{1}{2} \varpi: \widehat{M}_{x}+\zeta(x) \widehat{Q}\right\} .
$$

\subsection{Expansion on thermal vorticity}

Following ref. [23] we use linear response theory to evaluate thermal expectation values in the case of constant electromagnetic field. The purpose of this section is to give the thermal expectation value of an operator $\widehat{O}$ at the point $x$ as a thermal vorticity expansion. Using the properties of the trace, we can transfer the $x$ dependence from the operator $\widehat{O}$ to the statistical operator (41) written around the same point $x$ :

$$
\begin{aligned}
\langle\widehat{O}(x)\rangle & =\frac{1}{Z} \operatorname{tr}\left[\exp \left\{-\beta(x) \cdot \widehat{\pi}_{x}+\frac{1}{2} \varpi: \widehat{M}_{x}+\zeta(x) \widehat{Q}\right\} \widehat{O}(x)\right] \\
& =\frac{1}{Z} \operatorname{tr}\left[\widehat{\mathrm{T}}(-x) \exp \left\{-\beta(x) \cdot \widehat{\pi}_{x}+\frac{1}{2} \varpi: \widehat{M}_{x}+\zeta(x) \widehat{Q}\right\} \widehat{\mathrm{T}}^{-1}(-x) \widehat{O}(0)\right] \\
& =\frac{1}{Z} \operatorname{tr}\left[\exp \left\{-\beta(x) \cdot \widehat{\pi}+\frac{1}{2} \varpi: \widehat{M}+\zeta(x) \widehat{Q}\right\} \widehat{O}(0)\right] .
\end{aligned}
$$

To evaluate the mean value we expand the statistical operator of the last equality around vanishing vorticity. First, we split the exponent of the statistical operator in two parts as follows:

$$
\widehat{\rho}=\frac{1}{Z} \exp [\widehat{A}+\widehat{B}], \quad \widehat{A} \equiv-\beta_{\mu}(x) \widehat{\pi}^{\mu}+\zeta(x) \widehat{Q}, \quad \widehat{B} \equiv \frac{1}{2} \varpi: \widehat{M},
$$

and then we expand on $\widehat{B}$, which is the part containing thermal vorticity. Since $\widehat{B}$ and $\widehat{A}$ satisfy the same algebra of the case discussed in [23], the expansion will leads to the same result, which is 


$$
\begin{aligned}
\langle\widehat{O}(x)\rangle= & \langle\widehat{O}(0)\rangle_{\beta(x)}-\alpha_{\rho}\left\langle\left\langle\widehat{K}^{\rho} \widehat{O}\right\rangle\right\rangle-w_{\rho}\left\langle\left\langle\widehat{J}^{\rho} \widehat{O}\right\rangle\right\rangle+\frac{\alpha_{\rho} \alpha_{\sigma}}{2}\left\langle\left\langle\widehat{K}^{\rho} \widehat{K}^{\sigma} \widehat{O}\right\rangle\right\rangle \\
& +\frac{w_{\rho} w_{\sigma}}{2}\left\langle\left\langle\widehat{J}^{\rho} \widehat{J}^{\sigma} \widehat{O}\right\rangle\right\rangle+\frac{\alpha_{\rho} w_{\sigma}}{2}\left\langle\left\langle\left\{\widehat{K}^{\rho}, \widehat{J}^{\sigma}\right\} \widehat{O}\right\rangle\right\rangle+O\left(\varpi^{3}\right),
\end{aligned}
$$

where we defined

$$
\begin{aligned}
\left\langle\left\langle\widehat{K}^{\rho_{1}} \cdots \widehat{K}^{\rho_{n}} \widehat{J}^{\sigma_{1}} \cdots \widehat{J}^{\sigma_{m}}\right.\right. & \widehat{O}\rangle\rangle \equiv \int_{0}^{|\beta|} \frac{\mathrm{d} \tau_{1} \cdots \mathrm{d} \tau_{n+m}}{|\beta|^{n+m}} \times \\
& \times\left\langle\mathrm{T}_{\tau}\left(\widehat{K}_{-\mathrm{i} \tau_{1} u}^{\rho_{1}} \cdots \widehat{K}_{-\mathrm{i} \tau_{n} u}^{\rho_{n}} \widehat{J}_{-\mathrm{i} \tau_{n+1} u}^{\sigma_{1}} \cdots \widehat{J}_{-\mathrm{i} \tau_{n+m} u}^{\sigma_{m}} \widehat{O}(0)\right)\right\rangle_{\beta(x), c} .
\end{aligned}
$$

As discussed in Sec. 3.1, the boost and rotation defined starting from $\widehat{M}^{\mu v}$, i.e.

$$
\widehat{K}^{\mu}=u_{\lambda} \widehat{M}^{\lambda \mu}, \quad \widehat{J}^{\mu}=\frac{1}{2} \epsilon^{\alpha \beta \gamma \mu} u_{\alpha} \widehat{M}_{\beta \gamma},
$$

are different from those of a system without external electromagnetic field. The other difference with [23] is that in this case the averages $\langle\cdots\rangle_{\beta(x)}$ are made with the statistical operator

$$
\widehat{\rho}_{0}=\frac{1}{Z_{0}} \exp \{-\beta(x) \cdot \widehat{\pi}+\zeta(x) \widehat{Q}\} .
$$

\subsection{Currents and chiral anomaly}

In this section we determine the constitutive equations for the electric and the axial current at first order in thermal vorticity and we investigate the contributions from electric and magnetic fields to the thermal coefficients related to vorticity. The constitutive equations are obtained from the expansion on thermal vorticity given in the previous section. Instead of a direct evaluation we use the conservation equations to show that indeed no additional corrections from electric and magnetic field occur to first order vorticous coefficients, such as the conductivity of the Chiral Vortical Effect (CVE) and of the Axial Vortical Effect (AVE). In this way we obtain several relations between those coefficients and their relation to the chiral anomaly.

Consider the case of global thermal equilibrium with constant vorticity $\varpi_{\mu \nu}$ and an electromagnetic field with strength tensor $F_{\mu \nu}=k \varpi_{\mu \nu}$, with $k$ a constant. It then follows that the comoving magnetic and electric fields are parallel respectively to thermal rotation and thermal acceleration:

$$
B^{\mu}(x)=-k w^{\mu}(x), \quad E^{\mu}(x)=k \alpha^{\mu}(x) .
$$

For instance, in the case of a constant thermal vorticity caused by a rigid rotation along the $z$ axis and a constant magnetic field along $z$, we have:

$$
\varpi_{\mu \nu}=\frac{\Omega}{T_{0}}\left(\eta_{\mu 1} \eta_{\nu 2}-\eta_{\nu 1} \eta_{\mu 2}\right), \quad F_{\mu \nu}=B\left(\eta_{\mu 1} \eta_{\nu 2}-\eta_{\nu 1} \eta_{\mu 2}\right), \quad k=\frac{B T_{0}}{\Omega},
$$


where $\Omega, T_{0}$ and $B$ are constants. In this example, electric and magnetic fields are orthogonal and there is no chiral anomaly. However, in the general case, the product $E \cdot B$ is non-vanishing. In that case, as we showed in Sec. 2, we can still discuss global equilibrium with chiral imbalance by defining a conserved Chern-Simons current.

By using the thermal vorticity expansion (42) we now proceed to write the thermal expectation value of electric current at first order in thermal vorticity. We want to stress that in the expansion (42) no approximations are made on the effects of the external electric and magnetic fields; the expansion (42) only approximates the effects of vorticity. At first order on thermal vorticity the only quantities that can contribute to the mean value of a current are the four-vectors $w^{\mu}, \alpha^{\mu}$ and the scalars $E \cdot \alpha, E \cdot w=-B \cdot \alpha$ and $B \cdot w$. We therefore write the thermal expansion in terms of these quantities, which will define several thermal coefficients. Taking into account the symmetries, the thermal vorticity expansion of the electric current is

$$
\begin{aligned}
\left\langle\widehat{j}^{\mu}(x)\right\rangle= & {\left[n_{\mathrm{c}}^{0}+n_{\mathrm{c}}^{E \cdot \alpha}(E \cdot \alpha)+n_{\mathrm{c}}^{B \cdot w}(B \cdot w)\right] u^{\mu}+W^{\mathrm{V}} w^{\mu}+\sigma_{E}^{B \cdot \alpha}(B \cdot \alpha) E^{\mu} } \\
& +\left[\sigma_{B}^{0}+\sigma_{B}^{E \cdot \alpha}(E \cdot \alpha)+\sigma_{B}^{B \cdot w}(B \cdot w)\right] B^{\mu}+O\left(\varpi^{2}\right) .
\end{aligned}
$$

Since the thermal coefficients $n_{\mathrm{c}}^{0}$ and $\sigma_{B}^{0}$ must be evaluated at vanishing thermal vorticity, they are exactly those computed in Sec. 4.4 (for vanishing electric field). In particular, the Chiral Magnetic Effect (CME) conductivity at vanishing vorticity, Eq. (37), is

$$
\sigma_{B}^{0}(x)=\frac{q \zeta_{A}}{2 \pi^{2}|\beta(x)|}
$$

All the other coefficients are related to thermal vorticity and they have the following properties under parity, time-reversal and charge conjugation

\begin{tabular}{cccc|ccccccc}
\multicolumn{2}{c}{$E \cdot \alpha$} & $E \cdot w$ & $B \cdot w$ & $n_{\mathrm{c}}^{0}$ & $\sigma_{B}^{0}$ & $W^{\mathrm{V}}$ & $\sigma_{E}^{0}$ & $\sigma_{B}^{E \cdot \alpha}$ & $\sigma_{B}^{B \cdot w}$ & $\sigma_{E}^{B \cdot \alpha}$ \\
\hline $\mathrm{P}+$ & + & - & + & + & - & - & + & - & - & - \\
$\mathrm{T}+$ & - & + & + & + & + & - & + & + & + \\
$\mathrm{C}$ & - & - & - & - & + & - & + & - & - & -
\end{tabular}

Similarly the axial current thermal expectation value is

$$
\begin{aligned}
\left\langle\widehat{j}_{\mathrm{A}}^{\mu}(x)\right\rangle= & {\left[n_{\mathrm{A}}^{0}+n_{\mathrm{A}}^{E \cdot \alpha}(E \cdot \alpha)+n_{\mathrm{A}}^{B \cdot w}(B \cdot w)\right] u^{\mu}+W^{\mathrm{A}} w^{\mu}+\sigma_{s E}^{B \cdot \alpha}(B \cdot \alpha) E^{\mu} } \\
& +\left[\sigma_{s}^{0}+\sigma_{s}^{E \cdot \alpha}(E \cdot \alpha)+\sigma_{s}^{B \cdot w}(B \cdot w)\right] B^{\mu}+O\left(\varpi^{2}\right) .
\end{aligned}
$$

Each thermal coefficients is a function depending only on

$$
|\beta|, \zeta, \zeta_{\mathrm{A}}, B^{2}, E^{2}, E \cdot B .
$$

The coordinate dependence of any thermal coefficients is completely contained inside the Lorentz scalars in (46).

With the constitutive equations written down, we are now looking for relations and constraints between those thermodynamic coefficients. The conservation of electric 
current implies that

$$
\partial_{\mu}\left\langle\widehat{j}^{\mu}(x)\right\rangle=\left\langle\partial_{\mu} \widehat{j}^{\mu}(x)\right\rangle=0 .
$$

The coordinate derivative acts both on thermal coefficients and on thermodynamic fields. We need to establish how the derivative acts on those quantities. For thermodynamic fields, using the equilibrium conditions and the identities in Appendix 5.2, we find

$$
\begin{aligned}
\partial_{\mu} u^{\mu} & =0, \quad \partial_{\mu} w^{\mu}=-3 \frac{w \cdot \alpha}{|\beta|}, \quad \partial_{\mu} \alpha^{\mu}=\frac{2 w^{2}-\alpha^{2}}{|\beta|}, \\
\partial_{\mu} B^{\mu} & =-3 \frac{B \cdot \alpha}{|\beta|}, \quad \partial_{\mu} E^{\mu}=-\frac{2(w \cdot B)+(\alpha \cdot E)}{|\beta|}, \quad \partial_{\mu}(B \cdot \alpha)=0, \\
\partial_{\mu}(E \cdot \alpha) & =-\frac{2}{|\beta|}\left[(w \cdot B) \alpha_{\mu}+(E \cdot w) w_{\mu}\right], \\
\partial_{\mu}(B \cdot w) & =\frac{2}{|\beta|}\left[(w \cdot B) \alpha_{\mu}+(E \cdot w) w_{\mu}\right] .
\end{aligned}
$$

Moreover, we can also show that

$$
\begin{array}{r}
\partial_{\mu}|\beta|=-\alpha_{\mu}, \quad \partial_{\mu} \zeta=\beta^{v} F_{\nu \mu}=-|\beta| E_{\mu}, \quad \partial_{\mu} \zeta_{\mathrm{A}}=0, \quad \partial_{\mu}(E \cdot B)=0, \\
\partial_{\mu}|B|=-\frac{(E \cdot B) w_{\mu}+B^{2} \alpha_{\mu}}{|\beta||B|}, \quad \partial_{\mu}|E|=-\frac{(E \cdot B) w_{\mu}+B^{2} \alpha_{\mu}}{|\beta||E|},
\end{array}
$$

where

$$
|\beta|=\sqrt{\beta^{\sigma} \beta_{\sigma}}, \quad|B|=\sqrt{-B^{\sigma} B_{\sigma}}, \quad|E|=\sqrt{-E^{\sigma} E_{\sigma}} .
$$

The derivative respect to coordinates of a thermodynamic function is

$$
\begin{aligned}
\partial_{\mu} f\left(|\beta|, \zeta, \zeta_{A},|B|,|E|, E \cdot B\right)= & \left(-\partial_{\mu}|\beta| \frac{\partial}{\partial|\beta|}+\partial_{\mu} \zeta \frac{\partial}{\partial \zeta}+\partial_{\mu} \zeta_{\mathrm{A}} \frac{\partial}{\partial \zeta_{\mathrm{A}}}+\partial_{\mu}|B| \frac{\partial}{\partial|B|}\right. \\
& \left.+\partial_{\mu}|E| \frac{\partial}{\partial|E|}+\partial_{\mu}(E \cdot B) \frac{\partial}{\partial(E \cdot B)}\right) f .
\end{aligned}
$$

Therefore, using the previous identities, the derivative of a thermodynamic function becomes

$$
\begin{aligned}
\partial_{\mu} f= & {\left[-\alpha_{\mu}\left(\frac{\partial}{\partial|\beta|}-\frac{|B|^{2}}{|\beta|} \frac{1}{|B|} \frac{\partial}{\partial|B|}-\frac{|B|^{2}}{|\beta|} \frac{1}{|E|} \frac{\partial}{\partial|E|}\right)\right.} \\
& \left.-|\beta| E_{\mu} \frac{\partial}{\partial \zeta}-\frac{(E \cdot B) w_{\mu}}{|\beta|}\left(\frac{1}{|B|} \frac{\partial}{\partial|B|}+\frac{1}{|E|} \frac{\partial}{\partial|E|}\right)\right] f .
\end{aligned}
$$

We can also define the following short-hand notation:

$$
\partial_{\tilde{\beta}} \equiv \frac{\partial}{\partial|\beta|}-\frac{|B|^{2}}{|\beta|} \partial_{\tilde{B}}, \quad \partial_{\tilde{B}} \equiv \frac{1}{|B|} \frac{\partial}{\partial|B|}+\frac{1}{|E|} \frac{\partial}{\partial|E|},
$$

from which the previous derivative is written as 


$$
\partial_{\mu} f=\left[-\alpha_{\mu} \partial_{\tilde{\beta}}-|\beta| E_{\mu} \partial_{\zeta}-\frac{(E \cdot B) w_{\mu}}{|\beta|} \partial_{\tilde{B}}\right] f .
$$

We can now use the previous relations to impose electric current conservation by evaluating the divergence of the expansion in Eq. (43). For the terms directed along the fluid velocity we find that no additional constraints are required:

$$
\partial_{\mu}\left(n^{0} u^{\mu}\right)=\partial_{\mu}\left(n^{E \cdot \alpha}(E \cdot \alpha) u^{\mu}\right)=\partial_{\mu}\left(n^{B \cdot w}(B \cdot w) u^{\mu}\right)=0 .
$$

For the terms along the magnetic field we find:

$$
\begin{aligned}
\partial_{\mu}\left(\sigma_{B}^{0} B^{\mu}\right)= & -(B \cdot \alpha)\left[\frac{3}{|\beta|}+\partial_{\tilde{\beta}}\right] \sigma_{B}^{0}-(E \cdot B)|\beta| \partial_{\zeta} \sigma_{B}^{0} \\
& -\frac{(E \cdot B)(B \cdot w)}{|\beta|} \partial_{\tilde{B}} \sigma_{B}^{0} \\
\partial_{\mu}\left(\sigma_{B}^{E \cdot \alpha}(E \cdot \alpha) B^{\mu}\right)= & -(B \cdot \alpha)(E \cdot \alpha)\left[\frac{3}{|\beta|}+\partial_{\tilde{\beta}}\right] \sigma_{B}^{E \cdot \alpha}-(E \cdot \alpha)(E \cdot B)|\beta| \partial_{\zeta} \sigma_{B}^{E \cdot \alpha} \\
& -\frac{(E \cdot B)(B \cdot w)(E \cdot \alpha)}{|\beta|} \partial_{\tilde{B}} \sigma_{B}^{E \cdot \alpha} \\
& -\frac{2}{|\beta|}[(w \cdot B)(B \cdot \alpha)+(E \cdot w)(B \cdot w)] \sigma_{B}^{E \cdot \alpha}, \\
\partial_{\mu}\left(\sigma_{B}^{B \cdot w}(B \cdot w) B^{\mu}\right)= & -(B \cdot \alpha)(B \cdot w)\left[\frac{3}{|\beta|}+\partial_{\tilde{\beta}}\right] \sigma_{B}^{B \cdot w}-(B \cdot w)(E \cdot B)|\beta| \partial_{\zeta} \sigma_{B}^{B \cdot w} \\
& -\frac{(E \cdot B)(B \cdot w)^{2}}{|\beta|} \partial_{\tilde{B}} \sigma_{B}^{B \cdot w} \\
& +\frac{2}{|\beta|}[(w \cdot B)(B \cdot \alpha)+(E \cdot w)(B \cdot w)] \sigma_{B}^{B \cdot w} .
\end{aligned}
$$

Along electric field we have

$$
\begin{aligned}
\partial_{\mu}\left(\sigma_{E}(B \cdot \alpha) E^{\mu}\right)= & -(B \cdot \alpha)(\alpha \cdot E)\left[\frac{1}{|\beta|}+\partial_{\tilde{\beta}}\right] \sigma_{E}-(B \cdot \alpha) E^{2}|\beta| \partial_{\zeta} \sigma_{E} \\
& -\frac{(E \cdot B)(E \cdot w)}{|\beta|} \partial_{\tilde{B}} \sigma_{E} .
\end{aligned}
$$

Lastly, the divergence of the term along rotation is

$$
\partial_{\mu}\left(W^{\mathrm{V}} w^{\mu}\right)=-(w \cdot \alpha)\left[\frac{3}{|\beta|}+\partial_{\tilde{\beta}}\right] W^{\mathrm{V}}-(E \cdot w)|\beta| \partial_{\zeta} W^{\mathrm{V}}-\frac{(E \cdot B) w^{2}}{|\beta|} \partial_{\tilde{B}} W^{\mathrm{V}} .
$$

To impose that $\partial_{\mu}\left\langle\widehat{j}^{\mu}(x)\right\rangle=0$ we sum all the previous pieces and we split between the linear independent terms. Those terms must vanish independently of the values of the electromagnetic field and of the thermal vorticity and several equalities are obtained. Among those, we first consider the following identities: 
Thermal chiral fermions with vorticity and magnetic field

$$
\begin{aligned}
\partial_{\zeta} \sigma_{B}^{0}=0, & \partial_{\tilde{B}} W^{\mathrm{V}}=0, \quad \partial_{\zeta} \sigma_{B}^{E \cdot \alpha}=0, \quad \partial_{\tilde{B}} \sigma_{B}^{E \cdot \alpha}=0, \quad \partial_{\tilde{B}} \sigma_{B}^{B \cdot w}=0, \\
\partial_{\zeta} \sigma_{E}^{B \cdot \alpha}=0, & \partial_{\tilde{B}} \sigma_{E}^{B \cdot \alpha}=0 .
\end{aligned}
$$

Notice from the table in (45) that $\sigma_{B}^{E \cdot \alpha}$ and $\sigma_{E}^{B \cdot \alpha}$ are related to C-odd correlator. Therefore they must be odd functions of the electric chemical potential $\zeta$. But the previous constraints require that they do not depend on $\zeta$, therefore they must be vanishing

$$
\sigma_{B}^{E \cdot \alpha}=0, \quad \sigma_{E}^{B \cdot \alpha}=0
$$

The previous constraints also require that $W^{\mathrm{V}}$ and $\sigma_{B}^{B \cdot w}$ do not depend on $|B|$ and $|E|^{3}$. That is to say that the CVE conductivity $W^{\mathrm{V}}$ is not affected by electric and magnetic field. Moreover, electric current conservation also imposes that

$$
\begin{aligned}
\left(3+|\beta| \partial_{\tilde{\beta}}\right) \sigma_{B}^{0}-|\beta|^{2} \partial_{\zeta} W^{\mathrm{V}} & =0 \\
\partial_{\tilde{B}} \sigma_{B}^{0}+|\beta|^{2} \partial_{\zeta} \sigma_{B}^{B \cdot w} & =0 \\
\left(3+|\beta| \partial_{\tilde{\beta}}\right) \sigma_{B}^{B \cdot w} & =0 \\
\left(3+|\beta| \partial_{\beta}\right) W^{\mathrm{V}} & =0
\end{aligned}
$$

We can replace the known result for the CME conductivity $\sigma_{B}^{0}$ of Eq. (44) in the previous constraints to find find that

$$
\begin{aligned}
\partial_{\zeta} W^{\mathrm{V}} & =\frac{1}{|\beta|^{2}}\left(3+|\beta| \partial_{\tilde{\beta}}\right) \sigma_{B}^{0}=\frac{q \zeta_{\mathrm{A}}}{\pi^{2}|\beta|^{3}}, \\
\partial_{\zeta} \sigma_{B}^{B \cdot w} & =0, \\
\left(3+|\beta| \frac{\partial}{\partial \beta}\right) W^{\mathrm{V}} & =0 .
\end{aligned}
$$

Again, since $\sigma_{B}^{B \cdot w}$ is C-odd it follows form second equation that it must be vanishing

$$
\sigma_{B}^{B \cdot w}=0 .
$$

We want to empathize that we have found a relation between CVE and CME conductivities:

$$
\partial_{\zeta} W^{\mathrm{V}}=\frac{1}{|\beta|^{2}}\left(3+|\beta| \partial_{\tilde{\beta}}\right) \sigma_{B}^{0}
$$

The CVE conductivity $W^{\mathrm{V}}$ in Eq. (22) satisfies this relation. It is important to notice that Eq. (47) completely determines the CVE conductivity from the CME one. Indeed, since $W^{\mathrm{V}}$ is odd under charge conjugation, by fixing the $\zeta$ part of $W^{\mathrm{V}}$ we obtain the entire coefficient. This also implies that the $\zeta$ part of $W^{\mathrm{V}}$ is dictated by the chiral anomaly as found in effective field theories [48]. Therefore, the CVE

${ }^{3}$ Note that to reduce the numbers of relations, we have indicated electric field and magnetic field derivative together with one derivative $\partial_{\tilde{B}}$. However, electric and magnetic fields are independent and each derivative must be considered independently. 
inherits all the properties proved for the CME. For instance, it is known that the CME conductivity is completely dictated by the chiral anomaly [49] and that it is protected from corrections coming from interactions [50, 51]. Since the relation (47) holds not only for a free theory but for any microscopic interactions, as long as global thermal equilibrium is concerned, then also the CVE conductivity is dictated by the chiral anomaly and it is universal. Despite the CVE can be related to the vector-axial anomalous term of vector current anomaly [52], Eq. (47) shows that it can be explained with just the electric charge conservation and the chiral anomaly.

Let us now move to the axial current. Similar steps can be followed to derive the constraint equations between the thermal coefficients of axial current. In this case we must impose the following identities between thermal expectation values:

$$
\partial_{\mu}\left\langle\widehat{j}_{\mathrm{A}}^{\mu}(x)\right\rangle=2 m\left\langle\mathrm{i} \bar{\Psi} \gamma^{5} \Psi\right\rangle-\frac{q^{2}(E \cdot B)}{2 \pi^{2}},
$$

where we also added the naive divergence term $2 m\left\langle i \overline{\mathrm{i}} \gamma^{5} \Psi\right\rangle$ which is due to the mass of the field. From symmetries the constitutive equation for the pseudo-scalar is

$$
\begin{aligned}
\left\langle\mathrm{i} \bar{\Psi} \gamma^{5} \Psi\right\rangle= & L^{E \cdot B}(E \cdot B)+L^{\alpha \cdot w}(\alpha \cdot w)+L^{E \cdot w}(E \cdot w) \\
& +L^{(E \cdot B) \alpha^{2}}(E \cdot B) \alpha^{2}+L^{(E \cdot B) w^{2}}(E \cdot B) w^{2} \\
& +L^{(E \cdot w)(E \cdot \alpha)}(E \cdot w)(E \cdot \alpha)+L^{(E \cdot w)(B \cdot w)}(E \cdot w)(B \cdot w)+O\left(\varpi^{3}\right) .
\end{aligned}
$$

The value of $L^{\alpha \cdot w}$ for free Dirac field has been reported in (20), and the other coefficients related to the electromagnetic field can be computed with Ritus method.

Because the axial current is not conserved we find different identities compared to the previous case of electric current. For instance, the identities related to the Chiral Separation Effect (CSE) conductivity $\sigma_{s}^{0}$ and to the AVE conductivity $W^{\mathrm{A}}$ are

$$
\begin{aligned}
\left(3+|\beta| \frac{\partial}{\partial \beta}\right) W^{\mathrm{A}} & =-2 m L^{\alpha \cdot w}, \\
\partial_{\zeta} \sigma_{s}^{0} & =\frac{q^{2}}{2 \pi^{2}|\beta|}-2 m L^{E \cdot B}, \\
\partial_{\tilde{B}} \sigma_{s}^{0} & =-|\beta|^{2} \partial_{\zeta} \sigma_{s}^{B \cdot w}, \\
\partial_{\tilde{B}} W^{\mathrm{A}} & =-2 m|\beta| L^{(E \cdot B) w^{2}}, \\
\partial_{\zeta} W^{\mathrm{A}} & =\frac{1}{|\beta|^{2}}\left(3+|\beta| \partial_{\tilde{\beta}}\right) \sigma_{s}^{0}-\frac{2 m}{|\beta|} L^{E \cdot w} .
\end{aligned}
$$

The first equation has been discussed in [23] and in Sec. 2.1. The second equation is similar to the first: the first term on the r.h.s. is coming from the chiral anomaly and the second from the naive anomaly. Therefore for a massive field, as discussed for the AVE, the CSE is not entirely dictated by the anomaly. It is then not surprising to find a corrections to the CSE [53] and that it is affected by the mass, see Eq. (38). In 
the massive case we also expect corrections from the external electromagnetic field both in the AVE and in the CSE conductivities.

On the other hand, for massless field those constraints becomes

$$
\begin{aligned}
\partial_{\zeta} \sigma_{s}^{0} & =\frac{q^{2}}{2 \pi^{2}|\beta|}, \\
\left(3+|\beta| \frac{\partial}{\partial \beta}\right) W^{\mathrm{A}} & =0, \quad \partial_{\tilde{B}} W^{\mathrm{A}}=0, \\
\partial_{\zeta} W^{\mathrm{A}} & =\frac{1}{|\beta|^{2}}\left(3+|\beta| \partial_{\beta}\right) \sigma_{s}^{0} .
\end{aligned}
$$

In this case the CSE conductivity is completely fixed by the chiral anomaly as it is clear by the first equation and by the fact that $\sigma_{s}^{0}$ must be an odd function of $\zeta$. For the symmetries of axial current, $W^{\mathrm{A}}$ has both terms which depend on $\zeta$ and terms which depend only on $\zeta_{\mathrm{A}}$ and $\beta$. All these terms must satisfy the equations in the second line. In particular we conclude that the AVE is not affected by the electromagnetic field. Moreover, from the third line we see that the terms related to $\zeta$ are fixed by the CSE conductivity and consequently they are dictated by the chiral anomaly. As it is evident from previous discussion, this only occurs for the massless field.

In summary, by imposing the conservation equation we conclude that, at global thermodynamic equilibrium with thermal vorticity and constant homogeneous electromagnetic field, the chiral vortical effect is dictated by the chiral magnetic effect. Then it is not affected by the mass of the particle, by the external electromagnetic field or by radiative corrections. For the axial current this analysis has showed that we need to distinguish between the massive and the massless case. In the latter case we found that the chiral anomaly completely fixes the whole chiral separation effect (CSE) but fixes only the part of axial vortical effect (AVE) conductivity which depends on the electric chemical potential. We also found that the AVE is not affected by the external electromagnetic field. For the massive case, despite it exists a relation between the CSE and the AVE, both of them are affected by the mass of the field, the external electromagnetic field and by radiative corrections.

Acknowledgements I carried out part of this work while visiting Stony Brook University (New York, U.S.A.). I would like to thank F. Becattini, E. Grossi and D. Kharzeev for stimulating discussions on the subject matter. This research was supported in part by the Florence University with the fellowship "Polarizzazione nei fluidi relativistici".

\section{Appendix: Thermodynamic relations in beta frame}

At global thermal equilibrium with thermal vorticity, thermodynamic fields satisfy several equilibrium relations which constraints their coordinate dependence. In the $\beta$ frame we can build several quantities from the four-vector $\beta$ and thermal vorticity $\varpi$ : 


$$
\begin{aligned}
& u_{\mu}=\frac{\beta_{\mu}}{\sqrt{\beta^{2}}} ; \quad \Delta^{\mu \nu}=g^{\mu \nu}-u^{\mu} u^{\nu} ; \quad \varpi_{\mu \nu}=\partial_{v} \beta_{\mu}=\epsilon_{\mu \nu \rho \sigma} w^{\rho} u^{\sigma}+\alpha_{\mu} u_{v}-\alpha_{\nu} u_{\mu} ; \\
& \alpha_{\mu}=\varpi_{\mu \nu} u^{\nu} ; \quad w_{\mu}=-\frac{1}{2} \epsilon_{\mu \nu \rho \sigma} \varpi^{\nu \rho} u^{\sigma} ; \quad \gamma_{\mu}=(\alpha \cdot \varpi)^{\lambda} \Delta_{\lambda \mu}=\epsilon_{\mu \nu \rho \sigma} w^{v} \alpha^{\rho} u^{\sigma} .
\end{aligned}
$$

Most of these quantities depend on coordinates and their derivatives are [33]:

$$
\begin{gathered}
\partial_{\nu} \beta_{\mu}=\varpi_{\mu \nu} ; \quad \partial_{\nu}=-\alpha_{v} \frac{\partial}{\partial \sqrt{\beta^{2}}} ; \quad \varpi: \varpi=2\left(\alpha^{2}-w^{2}\right) \\
\partial_{\nu} u_{\mu}=\frac{1}{\sqrt{\beta^{2}}}\left(\varpi_{\mu \nu}+\alpha_{\nu} u_{\mu}\right) ; \quad \partial^{\alpha} u_{\alpha}=0 ; \quad u_{\alpha} \partial^{\alpha} u_{\mu}=\frac{\alpha_{\mu}}{\sqrt{\beta^{2}}} ; \\
\partial_{\mu} \alpha_{v}=\frac{1}{\sqrt{\beta^{2}}}\left(\varpi_{\nu \rho} \varpi_{\mu}^{\rho}+\alpha_{\mu} \alpha_{\nu}\right) ; \quad \partial^{\alpha} \alpha_{\alpha}=\frac{1}{\sqrt{\beta^{2}}}\left(2 w^{2}-\alpha^{2}\right) ; \quad u_{\alpha} \partial^{\alpha} \alpha^{2}=0 ; \\
\partial_{\mu} w_{\nu}=\frac{1}{\sqrt{\beta^{2}}}\left(\alpha_{\mu} w_{\nu}-\frac{1}{2} \epsilon_{\nu \rho \sigma \lambda} \varpi^{\rho \sigma} \varpi_{\mu}^{\lambda}\right) ; \quad \partial^{\alpha} w_{\alpha}=-3 \frac{w \cdot \alpha}{\sqrt{\beta^{2}}} ; \quad u_{\alpha} \partial^{\alpha} w^{2}=0 ; \\
\alpha^{\sigma} \partial_{\mu} \alpha_{\sigma}=w^{\sigma} \partial_{\mu} w_{\sigma}=\frac{1}{\sqrt{\beta^{2}}}\left(w^{2} \alpha_{\mu}-(\alpha \cdot w) w_{\mu}\right) ; \quad \partial_{\mu}(\alpha \cdot w)=0 ; \\
\partial_{\alpha} \gamma^{\alpha}=0 ; \quad \partial^{\alpha} \Delta_{\alpha \beta}=-\frac{\alpha_{\beta}}{\sqrt{\beta^{2}}} .
\end{gathered}
$$

\section{References}

1. L. Adamczyk, et al., Nature 548, 62 (2017). DOI 10.1038/nature23004

2. D.E. Kharzeev, J. Liao, S.A. Voloshin, G. Wang, Prog. Part. Nucl. Phys. 88, 1 (2016). DOI 10.1016/j.ppnp.2016.01.001

3. D.E. Kharzeev, L.D. McLerran, H.J. Warringa, Nucl. Phys. A 803, 227 (2008). DOI 10.1016/ j.nuclphysa.2008.02.298

4. K. Fukushima, D.E. Kharzeev, H.J. Warringa, Phys. Rev. D78, 074033 (2008). DOI 10.1103/ PhysRevD.78.074033

5. U. Heinz, R. Snellings, Ann. Rev. Nucl. Part. Sci. 63, 123 (2013). DOI 10.1146/ annurev-nucl-102212-170540

6. R. Hakim, Introduction to Relativistic Statistical Mechanics: Classical and Quantum (World Scientific Publishing Co Inc, 2011)

7. W. Israel, Gen. Rel. Grav. 9, 451 (1978). DOI 10.1007/BF00759845

8. V. Canuto, H.Y. Chiu, Phys. Rev. 173, 1220 (1968). DOI 10.1103/PhysRev. 173.1220

9. X.G. Huang, A. Sedrakian, D.H. Rischke, Annals Phys. 326, 3075 (2011). DOI 10.1016/j.aop. 2011.08.001

10. P. Kovtun, JHEP 07, 028 (2016). DOI 10.1007/JHEP07(2016)028

11. N. Weickgenannt, X.L. Sheng, E. Speranza, Q. Wang, D.H. Rischke, Phys. Rev. D100, 056018 (2019). DOI 10.1103/PhysRevD.100.056018

12. J.H. Gao, Z.T. Liang, Phys. Rev. D100, 056021 (2019). DOI 10.1103/PhysRevD.100.056021

13. J.W. Chen, S. Pu, Q. Wang, X.N. Wang, Phys. Rev. Lett. 110(26), 262301 (2013). DOI 10.1103/PhysRevLett.110.262301

14. D.N. Zubarev, A.V. Prozorkevich, S.A. Smolyanskii, Theoretical and Mathematical Physics 40(3), 821 (1979). DOI 10.1007/BF01032069 
15. C.G. van Weert, Annals of Physics 140, 133 (1982). DOI 10.1016/0003-4916(82)90338-4

16. F. Becattini, L. Bucciantini, E. Grossi, L. Tinti, Eur. Phys. J. C75(5), 191 (2015). DOI 10.1140/epjc/s10052-015-3384-y

17. T. Hayata, Y. Hidaka, T. Noumi, M. Hongo, Phys. Rev. D92(6), 065008 (2015). DOI 10.1103/ PhysRevD.92.065008

18. M. Hongo, Annals Phys. 383, 1 (2017). DOI 10.1016/j.aop.2017.04.004

19. F. Becattini, M. Buzzegoli, E. Grossi, Particles 2(2), 197 (2019). DOI 10.3390/ particles2020014

20. F. Becattini, W. Florkowski, E. Speranza, Phys. Lett. B789, 419 (2019). DOI 10.1016/j. physletb.2018.12.016

21. F. Becattini, Phys. Rev. Lett. 108, 244502 (2012). DOI 10.1103/PhysRevLett.108.244502

22. F. Becattini, E. Grossi, Phys. Rev. D92, 045037 (2015). DOI 10.1103/PhysRevD.92.045037

23. M. Buzzegoli, F. Becattini, JHEP 12, 002 (2018). DOI 10.1007/JHEP12(2018)002

24. K. Jensen, R. Loganayagam, A. Yarom, JHEP 05, 134 (2014). DOI 10.1007/JHEP05(2014)134

25. V.E. Ambruş, E. Winstanley, Phys. Lett. B734, 296 (2014). DOI 10.1016/j.physletb.2014.05. 031

26. V.E. Ambrus, E. Winstanley, arXiv: 1908.10244 (2019)

27. F. Becattini, M. Buzzegoli, A. Palermo, arXiv:2007.08249 (2020)

28. F. Becattini, Phys. Rev. D97(8), 085013 (2018). DOI 10.1103/PhysRevD.97.085013

29. F. Becattini, D. Rindori, Phys. Rev. D99(12), 125011 (2019). DOI 10.1103/PhysRevD.99. 125011

30. G.Y. Prokhorov, O.V. Teryaev, V.I. Zakharov, Particles 3(1), 1 (2020). DOI 10.3390/ particles3010001

31. G.Y. Prokhorov, O.V. Teryaev, V.I. Zakharov, Phys. Rev. D99(7), 071901 (2019). DOI 10.1103/PhysRevD.99.071901

32. G.Y. Prokhorov, O.V. Teryaev, V.I. Zakharov, JHEP 03, 137 (2020). DOI 10.1007/ JHEP03(2020)137

33. M. Buzzegoli, E. Grossi, F. Becattini, JHEP 10, 091 (2017). DOI 10.1007/JHEP07(2018)119, 10.1007/JHEP10(2017)091. [Erratum: JHEP07,119(2018)]

34. M. Hongo, Y. Hidaka, Particles 2(2), 261 (2019). DOI 10.3390/particles2020018

35. N. Landsman, C. van Weert, Physics Reports 145(3), 141 (1987). DOI http://dx.doi.org/10. 1016/0370-1573(87)90121-9

36. A. Flachi, K. Fukushima, Phys. Rev. D98(9), 096011 (2018). DOI 10.1103/PhysRevD.98. 096011

37. G. Prokhorov, O. Teryaev, Phys. Rev. D97(7), 076013 (2018). DOI 10.1103/PhysRevD.97. 076013

38. G. Prokhorov, O. Teryaev, V. Zakharov, Phys. Rev. D98(7), 071901 (2018). DOI 10.1103/ PhysRevD.98.071901

39. L.J. Tassie, H.A. Buchdahl, Austral. J. Phys. 17, 431 (1964). DOI 10.1071/PH640431

40. H.A. Buchdahl, L.J. Tassie, Austral. J. Phys. 18, 109 (1965). DOI 10.1071/PH650109

41. H. Bacry, P. Combe, J.L. Richard, Nuovo Cim. A67, 267 (1970). DOI 10.1007/BF02725178

42. V.I. Ritus, Annals Phys. 69, 555 (1972). DOI 10.1016/0003-4916(72)90191-1

43. C.N. Leung, S.Y. Wang, Nucl. Phys. B747, 266 (2006). DOI 10.1016/j.nuclphysb.2006.04.028

44. M. Laine, A. Vuorinen, Lect. Notes Phys. 925, pp.1 (2016). DOI 10.1007/978-3-319-31933-9

45. K. Fukushima, D.E. Kharzeev, H.J. Warringa, Nucl. Phys. A836, 311 (2010). DOI 10.1016/j. nuclphysa.2010.02.003

46. A. Vilenkin, Phys. Rev. D22, 3080 (1980). DOI 10.1103/PhysRevD.22.3080

47. M.A. Metlitski, A.R. Zhitnitsky, Phys. Rev. D72, 045011 (2005). DOI 10.1103/PhysRevD.72. 045011

48. A. Sadofyev, V. Shevchenko, V. Zakharov, Phys. Rev. D 83, 105025 (2011). DOI 10.1103/ PhysRevD.83.105025

49. D.E. Kharzeev, Prog. Part. Nucl. Phys. 75, 133 (2014). DOI 10.1016/j.ppnp.2014.01.002

50. V.I. Zakharov, Lect. Notes Phys. 871, 295 (2013). DOI 10.1007/978-3-642-37305-3_11

51. B. Feng, D.F. Hou, H.C. Ren, Phys. Rev. D99(3), 036010 (2019). DOI 10.1103/PhysRevD.99. 036010 
52. K. Landsteiner, E. Megias, F. Pena-Benitez, Lect. Notes Phys. 871, 433 (2013). DOI 10.1007/ 978-3-642-37305-3_17

53. E.V. Gorbar, V.A. Miransky, I.A. Shovkovy, X. Wang, Phys. Rev. D88(2), 025025 (2013). DOI 10.1103/PhysRevD.88.025025 